\title{
The mode of sucrose degradation in potato tubers determines the fate of assimilate utilization
}

\author{
Stephanus J. Ferreira and Uwe Sonnewald* \\ Biochemistry Division, Department Biology, Friedrich-Alexander-University Erlangen-Nuremberg, Erlangen, Germany
}

\section{Edited by:}

Alisdair Fernie, Max Planck Institute

for Plant Physiology, Germany

\section{Reviewed by:}

Alisdair Fernie, Max Planck Institute for Plant Physiology, Germany Wagner L. Araújo, Universidade Federal de Viçosa, Brazil

\section{*Correspondence:}

Uwe Sonnewald, Biochemistry Division, Department Biology,

Friedrich-Alexander-University Erlangen-Nuremberg, Staudtstrasse

5, Erlangen 91058, Germany.

e-mail: usonne@biologie.

uni-erlangen.de
Cytosolic (U-IN-2) or apoplasmic (U-IN-1) targeting of yeast invertase in potato tubers leads to a reduction in sucrose and an increase in glucose content, but specific phenotypical changes are dependent on the subcellular targeting of the enzyme. Cytosolic expression leads to a more severe phenotype with the most striking aspects being reduced starch content and increased respiration. Despite extensive research, the regulatory mechanisms leading to these changes remain obscure. Recent technological advancements regarding potato transcriptional and genomic research presented us with the opportunity to revisit these lines and perform detailed gene expression analysis, in combination with extensive metabolic profiling, to identify regulatory networks underlying the observed changes. Our results indicate that in both genotypes reduced UDP-glucose production is associated with a reduced expression of cell wall biosynthetic genes. In addition, U-IN-1 tubers are characterized by elevated expression of senescence-associated genes, coupled to reduced expression of genes related to photosynthesis and the cytoskeleton. We provide evidence that increased respiration, observed specifically in U-IN-2 tubers, might be due to sugar signaling via released trehalose-6-phosphate inhibition of the SnRK1 complex. In both genotypes, expression of the plastidic glucose-6-phosphate transporter (GPT) is significantly down-regulated. This leads to a shift in the cytosolic to plastidic glucose-6-phosphate ratio and hence might limit starch synthesis but also the oxidative pentose phosphate pathway. This might explain the observed changes in several additional plastid localized pathways, most notably reduced expression of fatty acid biosynthetic genes and an accumulation of shikimate. Interestingly, a strict negative correlation between invertase and GPT expression could be observed in a wide range of potato tubers. This reciprocal regulation may be part of a more general switch controlling energy versus storage metabolism, suggesting that the fate of assimilate utilization is coordinated at the level of sucrose degradation.

Keywords: starch, potato, invertase, genomics, transcriptome

\section{INTRODUCTION}

Starch biosynthesis, especially in potato tubers, has extensively been studied. Attempts to increase starch content of tubers either focused on increasing carbon assimilation in leaves, i.e., enhanced source capacity, or stimulating the ability of tubers to attract assimilates, i.e., increased sink strength. Based on the analysis of transgenic potato plants with reduced source capacity (Zrenner et al., 1996) it seemed unlikely that source capacity limits tuber and starch yield under various growth conditions, including experimental field tests. Therefore, most attempts concentrated on increasing assimilate flux towards starch biosynthesis by manipulating tuber metabolism. In this respect enhancing glucose-6-phosphate (G6P) and/or ATP uptake of amyloplasts resulted in a significant increase in starch accumulation of transgenic potato plants under greenhouse conditions (Flügge et al., 2011). Another approach which was followed was to increase the sucrose hydrolytic activity in potato tubers. In the plant kingdom there are only two enzyme activities able to degrade sucrose, sucrose synthase (Susy), yielding fructose and UDP-Glucose, and invertase, yielding fructose and glucose.
Sucrose synthase activity is believed to be confined to the cytosol with isoforms of invertase present in the cell wall (cell wall-bound invertase), cytosol (neutral invertase), and vacuole (vacuolar invertase; Barratt et al., 2009); and there is also evidence of a plastidial isoform (Vargas et al., 2008). In potato, as in other plant species, the enzyme predominantly degrading sucrose is dependent on the tissue and/or physiological state of the specific tissue. For instance, the switch from invertase degradation to Susy degradation is seen as a marker for the onset of tuberization (Viola et al., 2001). Analyzing Susy antisense plants revealed that starch accumulation is highly dependent on Susy activity (Zrenner et al., 1995). Interestingly, soluble acid invertase activity and expression is strongly up-regulated in Susy deficient potato tubers. Nevertheless, the increased invertase activity cannot complement for the loss of Susy activity. In physiological terms the onset of tuberization is, importantly, a change from energy metabolism where sucrose is used for growth, to storage metabolism, where sucrose is mainly converted to starch for storage. At the same time, there is also a switch from apoplasmic to symplasmic sucrose unloading (Viola et al., 2001). 
The mode of sucrose degradation, sucrolytic or hydrolytic, has been analyzed in great detail. Many factors contribute to which enzyme activity is predominantly used. Comparing a large number of plant species and tissues revealed a tentative correlation between the mode of sucrose degradation and the nature of sink tissues. Hydrolytic sucrose degradation is mostly correlated to metabolic sinks, while sucrolytic sucrose degradation correlates strongly with storage sinks. The reason for this is elusive. Major differences between both pathways are compartmentation and production of UDP-glucose or glucose which is specific for sucrolytic or hydrolytic sucrose degradation, respectively. While glucose content positively correlates with meristem activity and sensing mechanisms have been described (Smeekens et al., 2010), little is known about the role of UDP-glucose. The production of UDP-Glucose instead of glucose may be attractive in the sense that it saves one ATP unit, which would be needed to phosphorylate glucose via hexokinase. In a relatively energy scarce environment like a potato tuber this could be a contributing factor to the change. Whether this is of significant importance is debatable since one glucose unit would produce 32 units of ATP via glycolysis and aerobic respiration, questioning whether the saving of one ATP would be a reason for an alternative route. Anaerobic respiration, however, would produce only four units of ATP, and the manner of cleavage might be important under such conditions.

Another important process requiring UDP-glucose is cell wall biosynthesis. It has been shown in cotton that a specific isoform of Susy, embedded in the cellulose synthase complex, is responsible for channeling the UDP-glucose to the cellulose synthase complex (Amor et al., 1995). It is not known whether such a mechanism would exist in potato tubers where cellulose, and also hemi-cellulose which is also produced from UDP-glucose, would represent only a small flux compared to starch biosynthesis. The importance of Susy activity for normal cell wall biosynthesis is unclear. In for instance growing stolons, characterized by an active cell wall biosynthesis, invertase rather than Susy activity is dominant. Moreover, in Arabidopsis mutants lacking Susy activity in all tissues except the phloem, there was no effect on cellulose or cell wall composition (Barratt et al., 2009).

Despite these differences, the products of both, invertase and Susy, can be converted to glucose-6-phosphate, the form in which carbon is most likely imported to the plastid for starch biosynthesis (Zhang et al., 2008). There is evidence that glucose-1-phosphate is transported into the plastid via an unidentified transporter (Fettke et al., 2010), although it has not been proven in vivo. In earlier attempts to increase sink strength, yeast invertase was expressed in potato tubers, where it was targeted to either the cytosol or apoplasm. Apoplasmic (U-IN-1) and cytosolic (U-IN-2) expression led to very different results. Cytosolic expression led to large changes in metabolite levels, reduced starch and, importantly, to an increase in respiration. This increase in tuber respiration led to an acceleration of starch degradation during potato tuber storage. Apoplasmic expression led to an increase in tuber size and, under certain conditions, also tuber yield. This increase, however, was due to an increase in the water content, and there was no effect on the starch content (Sonnewald et al., 1997; Hajirezaei et al., 2000). In later studies on U-IN-2, aiming to improve the starch yield, it was argued that the endogenous rates of hexokinase are not sufficient to convert the produced glucose to glucose-6phosphate. To circumvent this problem a bacterial glucokinase was over-expressed in the U-IN-2 background. Surprisingly, this led to a further increase in the levels of glycolytic metabolism and respiration, further reducing starch levels (Trethewey et al., 1998). Thus, despite the extensive research on yeast invertase expressing plants, the reasons for the shift to respiration in U-IN-2 are still not clear. Furthermore, also it is puzzling that such an increase in respiration is not observed in U-IN-1.

Potato research has been greatly enhanced with the development of an extensive and well annotated microarray, POCI (Kloosterman et al., 2008; Ferreira et al., 2010; Hartmann et al., 2011) and the sequencing of the potato genome ( $\mathrm{Xu}$ et al., 2011). This presented us with the opportunity to revisit these transgenic lines and to do a detailed study at the transcriptional and genomic level. Recently studies of starch biosynthesis have shifted from identifying structural genes required for the process toward the identification of regulatory mechanisms controlling it. In broad terms it seems as though the major regulatory mechanisms controlling starch metabolism are at the transcriptional level, through protein complex formation, redox regulation, and phosphorylation of enzymes (for review see Kötting et al., 2010).

Thus, the aim of this work was to elucidate the metabolic pathways and regulatory signals responsible for the phenotypical changes in both genotypes, with special emphasis on the increase in tuber respiration at the expense of starch biosynthesis in U-IN-2. To this end gene expression analysis of growing U-IN-1 and UIN-2 tubers were conducted in combination with metabolic data analysis. Furthermore, the data was also combined with previous studies performed on U-IN-1 and U-IN-2.

\section{RESULTS AND DISCUSSION}

\section{GLOBAL ANALYSIS REVEALS THAT OVEREXPRESSION OF APOPLASMIC OR CYTOSOLIC INVERTASE LEADS TO VERY DIFFERENT METABOLITE AND TRANSCRIPT PATTERNS}

Potato plants were grown under greenhouse conditions for 8 weeks after which tuber samples were taken and immediately frozen in liquid nitrogen. It can be assumed that the tubers were still actively growing at this stage. For gene expression analysis, RNA extracted from these samples were hybridized to the Agilent 44k POCI array (Kloosterman et al., 2008), and data extracted using GeneSpring 11 software as describe in the Section "Materials and Methods." As a starting point for the analysis, the global relationship between the different genotypes, based on expression data, was determined. To this end a principle component analysis was performed using either the individual replicates, or the mean expression values of each genotype. We were able to separate the genotypes on a PCA using expression data, with the replicates of each genotype clearly clustering into different groups. This was also true using the mean values. These findings are in disagreement with earlier reports which stated that it was not possible to separate genotypes using transcript profiling (Urbanczyk-Wochniak et al., 2003). It should be mentioned that the platform used for the transcriptional analysis in that study was based on the closely related species Solanum lycopersicum, making the current study more sensitive, which is probably the reason for the better separation (Figure 1). 


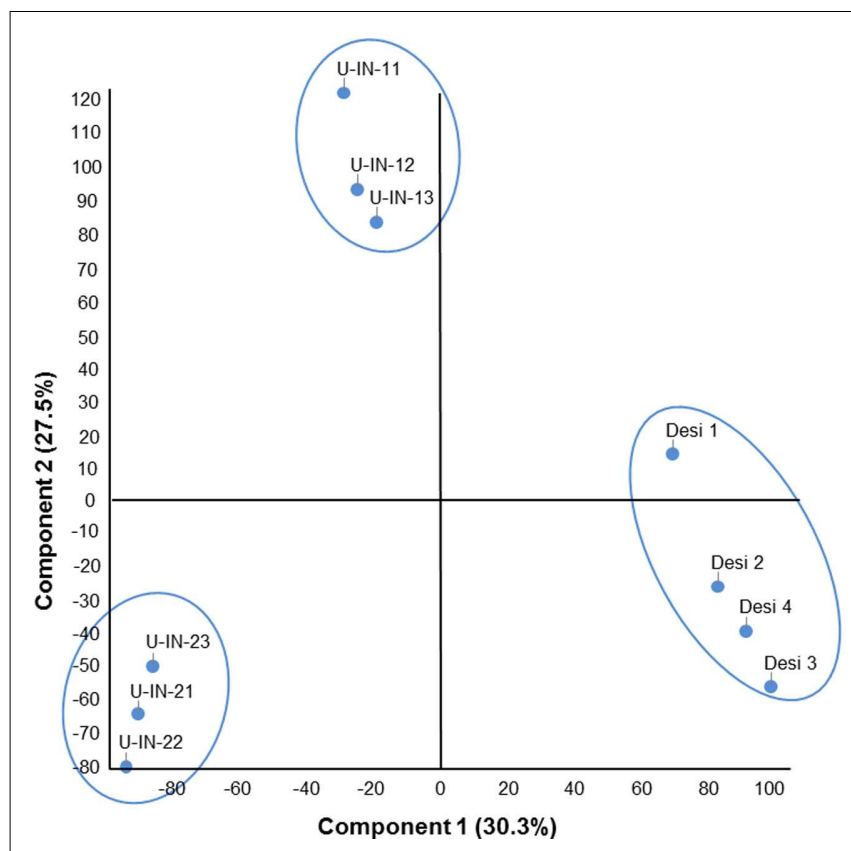

FIGURE 1 | Principle component analysis (PCA) of transcription data. Replicates illustrating that the individual replicates of each genotype clustered.

To determine which processes were mainly responsible for the observed phenotypes, a functional assignment of the features more than twofold differentially expressed compared to the wild type (WT) was performed. The functional assignment was performed according to functional groupings defined previously (Hartmann et al., 2011). For U-IN-2 there was no functional group more than twofold enriched amongst the up-regulated features. There was, however, a more than twofold enrichment in the down-regulated features for storage proteins, fatty acid metabolism, and cell wall metabolism.

U-IN-1, despite showing a milder phenotype than U-IN-2 and clustering closer to WT in the PCA, showed far more changes in terms of the number of functional groups changed. Of the upregulated features there was an enrichment of more than twofold in development and senescence, whilst redox/antioxidant, photosynthesis, fatty acid metabolism, cytoskeleton, and cell wall metabolism related features were enriched amongst the down-regulated features. The fact that photosynthetic transcripts were reduced is somewhat perplexing. A reason could of course be light contamination of the control tubers, but this is highly unlikely. Large pool sizes were used and no material from the tuber surface area was taken for RNA extractions. Metabolite analysis also did not reveal an increase in ribulose-1,5-bisphosphate, which could be seen as a marker metabolite for Calvin cycle activity (Table A1 in Appendix). Also, photosynthetic transcripts were not severely altered in U-IN-2, which was compared to the same control, indicating that the relative reduction in photosynthetic gene expression in U-IN-1 is not due to an increase in the control (Figure 2). It would indicate that genes associated with photosynthesis are expressed in heterotrophic tissue. This would not be unusual since several lines of evidence show that under certain conditions photosynthetic gene expression can be induced in heterotrophic tissue, for instance in sucrose starved Arabidopsis suspension cultures (Contento et al., 2004) and glutamate-1-semialdehyde aminotransferase silenced tomato fruit pericarp (Lytovchenko et al., 2011). A reason for the reduced expression in U-IN-1 could be the increase in glucose in the apoplasmic space, which is known to inhibit photosynthetic gene expression (Heineke et al., 1992). Other processes regulated in U-IN-1 could also be explained by the increase in apoplasmic glucose. For instance, glucose feeding of tobacco leaf disks lead to accelerated senescence (Wingler, 1998), suggesting that expression of senescence-associated genes might be regulated by sugar signals (for review see Wingler and Roitsch, 2008). This hypothesis is further supported by the observation, that silencing of cell wall invertase in tomato leaves resulted in a significantly increased sucrose to hexose ratio in the apoplasmic space and a delay in senescence (Kocal et al., 2008). In Arabidopsis it has been shown that senescence leads to the degradation of microtubules (Keech et al., 2010), explaining the reduction in gene expression associated with the cytoskeleton. It is worth mentioning that these changes were not observed in U-IN-2, providing evidence that the signal causing these changes must be extracellular.

A major factor which could have contributed to the differences observed between the genotypes would be the developmental state in which the tubers were at the time of harvesting. Although this cannot be ignored and might partially be responsible for observed changes, we believe that this would have only a minor influence on the results. The samples were taken at a relatively early stage during tuber development, 8 weeks after transferring the plants to the greenhouse. Since the B33 promoter driving the transgene expression only becomes active upon tuberization (Rocha-Sosa et al., 1989), the samples would have been taken not long after the activation of transgene expression and thus the tubers should be in similar developmental states.

Parallel to the transcriptional analysis, metabolic profiling of the genotypes was also performed (Tables A1-A4 in Appendix). PCA analysis showed that the genotypes could be separated and also provided clarity on which metabolites play the most significant role in the separation. Again, as with the transcripts, component one, responsible for $71 \%$ of the variance, was able to separate U-IN-2 from the other genotypes, whilst component two, responsible for $29 \%$ of the variance, could separate all three genotypes with U-IN-1 the furthest from the control (Figure 3). The metabolites most important for the variance in component one were sucrose, sucrose-6-phosphate (S6P), shikimate, and glucose. For component two the carotenoid violaxanthin, inorganic pyrophosphate, fructose-6-phosphate, and glucose had the biggest influence on the separation. Metabolic analysis also revealed no significant change in free amino acids (Table A2 in Appendix), which is in contrast to earlier studies on U-IN-2 (Trethewey et al., 1998; Farre et al., 2008). Possible reasons for this could be differences in growth and fertilization conditions. The time of sample taking would probably have a big effect on amino acid contents. As mentioned, respiration in U-IN-2 accelerates during storage, which would mean an increase in activity of glycolysis and the TCA cycle, leading to higher production of amino acids over time. Thus, the reason no increase in amino acid contents could be observed 

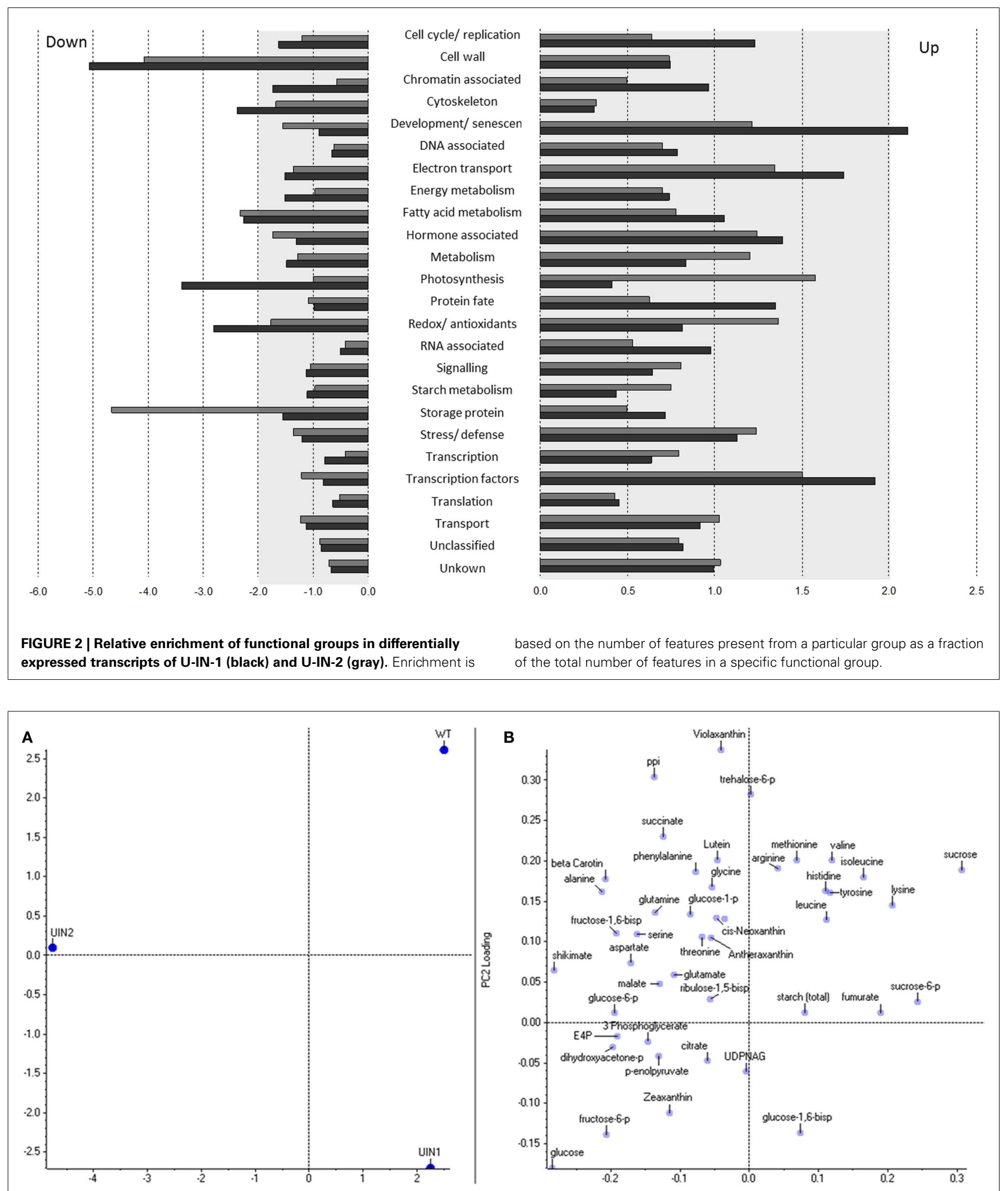

FIGURE 3 | Principle component analysis on metabolite levels. (A) The PCA separation of the genotypes and (B) the loadings of the individual metabolites. 
in contrast to previous studies, could be due to the relatively early time-point the samples were taken in the current study.

\section{REDUCED SUBSTRATE SUPPLY LIMITS CELL WALL AND FATTY ACID BIOSYNTHESIS IN BOTH GENOTYPES}

To further enhance our understanding of what happened in the transgenic lines, pathway specific analysis of transcripts and metabolites were performed using MapMan software (Thimm et al., 2004). The first pathways analyzed were cell wall and fatty acid biosynthesis, since global analysis revealed a down-regulation of transcripts associated with these in both U-IN-1 and U-IN2. Reduced expression of cell wall biosynthesis associated genes would be in line with reduced production of UDP-glucose by Susy. Using genomic (Xu et al., 2011) and expression data we could identify five differentially expressed isoforms of Susy with the dominant isoform in tubers, Susy 4 (Fu and Park, 1995; Zrenner et al., 1995; Ferreira et al., 2010), strongly down-regulated in U-IN-1. It did not show any change in expression in U-IN2. A striking aspect of Susy expression in U-IN-2 was the strong increase in expression of an isoform of Susy which is normally associated with stolons (Ferreira et al., 2010). It would be expected that cell wall biosynthesis is also active during stolon growth and meristematic activity, a time when Susy activity is low. It could well be that this isoform of Susy is active specifically under these conditions, producing the required UDP-glucose (Figure 4). This is probably not very successful considering the high invertase expression levels reached with the B33 promoter (Sonnewald et al., 1997; Hajirezaei et al., 2000), and invertase's much lower $K_{\mathrm{m}}$ for sucrose (Avigad, 1982). From the transcription data it seems quite clear, especially for U-IN-2, that the reactions requiring UDP-glucose are all down-regulated. There also seems to be other pathways activated in order to produce the required UDP-glucose and UDP-glucuronate. UDP-glucuronate can also be produced via a bypass reaction through the myo-inositol pathway. There was not a strong, uniform up-regulation of this pathway in either U-IN-1 or U-IN-2, although most transcripts of the rate-limiting enzyme in the pathway, Myo-inositol-1-phosphate synthase (MI1PS; Loewus and Loewus, 1980; Loewus et al., 1980) were strongly up-regulated, especially in U-IN-2. Glucose-6-phosphate can also be converted to UDP-glucose via cytosolic phosphoglucomutase (cPGM) and UDP-glucose pyrophosphorylase (UGPase). In both transgenic lines there was increased cPGM expression, and in U-IN-2 there was also an increase in the expression of UGPase transcripts (Figure 4).

As mentioned, global analysis indicated a reduction in fatty acid metabolism in both U-IN-1 and U-IN-2. Closer inspection of the metabolic path leading to fatty acid biosynthesis revealed striking similarity between U-IN-1 and U-IN-2. Plastidial AcetylCoA, substrate for fatty acid biosynthesis, is produced from PEP by plastidial pyruvate kinase and pyruvate dehydrogenase. It has been proven beyond reasonable doubt that all the Acetyl-CoA in the plastid is produced there, since it cannot be transported across the plastid membrane (Kohlhaw and Tan-Wilson, 1977; Patel and Clark, 1980; Rangasamy and Ratledge, 2000) Despite an increase in PEP, there was a reduction in the expression of plastidial pyruvate kinase and pyruvate dehydrogenase, indicating that the pool of PEP is not available for Acetyl-CoA synthesis in the plastid. There was no such reduction for plastidial pyruvate kinase in U-IN-1, the only major difference in expression between the two genotypes regarding fatty acid biosynthesis. Plastidial Acetyl-CoA can also be produced from citrate by ATP: citrate lyase, either in the cytosol or in the plastid from imported citrate, with the distribution of cytosolic to plastidial activity varying greatly between species (Rangasamy and Ratledge, 2000). Although it was difficult to distinguish between cytosolic and plastidial isoforms in our study, it was quite clear that there is a significant reduction in expression of most of the transcripts encoding the different isoforms of this gene. Furthermore, there was also a strong reduction in the expression of genes involved in subsequent steps of fatty acid biosynthesis (Figure 5).

\section{ALTHOUGH SPECIFIC CHANGES ARE ASSOCIATED WITH APOPLASMIC OR CYTOSOLIC INVERTASE, HIGH INVERTASE ACTIVITY SEEMINGLY REDUCES STARCH BIOSYNTHETIC CAPACITY}

Based on a global inspection of functional groups (Figure 2) there was no obvious change in gene expression related to starch metabolism. There was, however, a strong enrichment of storage protein related transcripts amongst the most-down-regulated features in U-IN-2, a process closely associated with starch accumulation (Zrenner et al., 1995), and metabolic data from this and previous studies show that there was a reduction in starch content in U-IN-2 (Table A3 in Appendix). For this reason, and the obvious importance of starch biosynthesis in tubers, starch metabolism, starting with the degradation of sucrose, and the subsequent phosphorylation of hexoses, was further investigated in U-IN-2 and U-IN-1 (Figure 6). Genomic alignment of EST sequences revealed three cell wall invertase genes differentially expressed. It was remarkable to note that despite the presence of a highly active transgenic invertase in the cell wall, the expression of endogenous invertases were up-regulated in U-IN-1. This was also the case for one isoform in U-IN-2. The regulation of invertase gene expression and activity differs greatly between isoforms (Roitsch and González, 2004), with some being activated through a feed-forward mechanism by glucose (Roitsch et al., 1995), which would explain the observed increase. It is however surprising that there was so much difference in the regulation between the two genotypes, showing that the response to glucose for some genes requires the signal to come from outside the cell, similar to what was discussed earlier.

Glucose-6-phosphate (G6P), the metabolite imported into the plastid for starch biosynthesis, can be produced from all three products of sucrose degradation. Under normal conditions, Susy activity is correlated to the rate of starch biosynthesis in potato tubers (Zrenner et al., 1995). Thus, G6P produced from fructose via fructokinase and phosphoglucose isomerase, and from UDP-Glucose, via UDP-Glucose pyrophosphorylase and cytosolic phosphoglucomutase, would be the primary route. In U-IN-1 and U-IN-2 reduced production of UDP-Glucose would mean that another pathway for starch biosynthesis should be activated, or at least increased, and that is via the phosphorylation of glucose by hexokinase. This is indeed the case with hexokinase 5 expression strongly increased in U-IN-2. Sequence similarity to hexokinase 5 from tobacco indicated that it is most probably bound to the mitochondrial membrane (Giese, 2004). Reduced expression of fructokinase 2 in U-IN-1, one of the most important isoforms 
A

$\left.\right|_{0} ^{1,3}$
-0.5
0
0.5
0.3

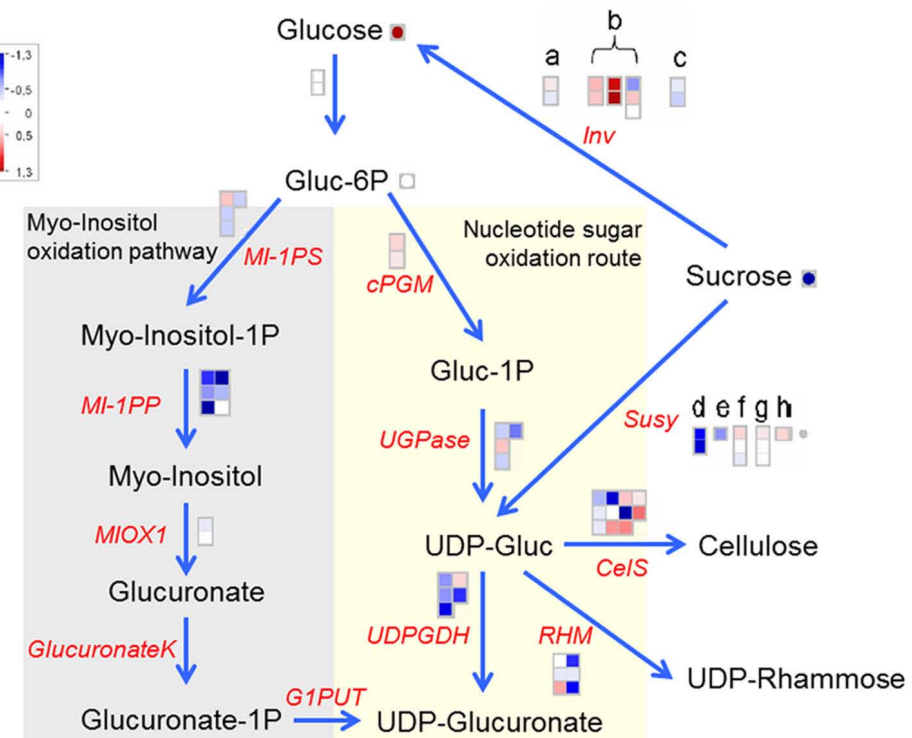

Glucuronate-1P $\stackrel{\text { G1PUT }}{\longrightarrow}$ UDP-Glucuronate

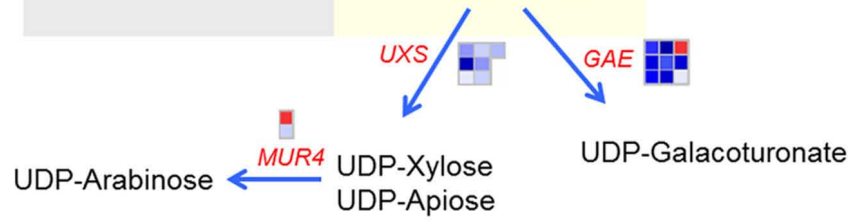

B

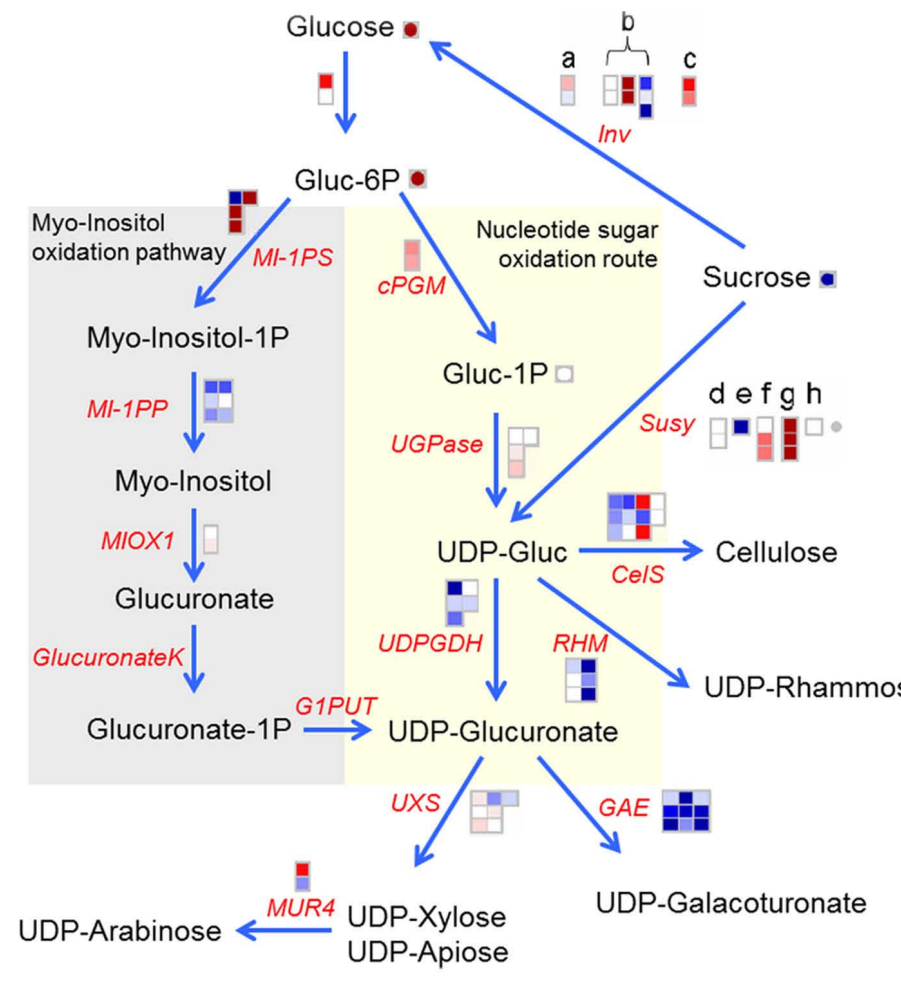

FIGURE 4 |The production of UDP-glucose and UDP-glucuronate for cell wall biosynthesis in (A) U-IN-1 and (B) U-IN-2. (a) Neutral invertase. (b) Cell wall invertase (c) Vacuolar invertase. (d-h) differentially expressed Susy Isoforms with (d) Susy4. Gene name abbreviations: MI-1PP,
Myo-inositol-1-phosphate phosphatase; Glucuronate K, glucuronate kinase; G1PUT, galactose-1-phosphate uridylyltransferase; UXS, UDP-xylose synthase; GAE, UDP-glucuronate epimerase; RHM, rhamnose synthase; CelS, cellulose synthase. 

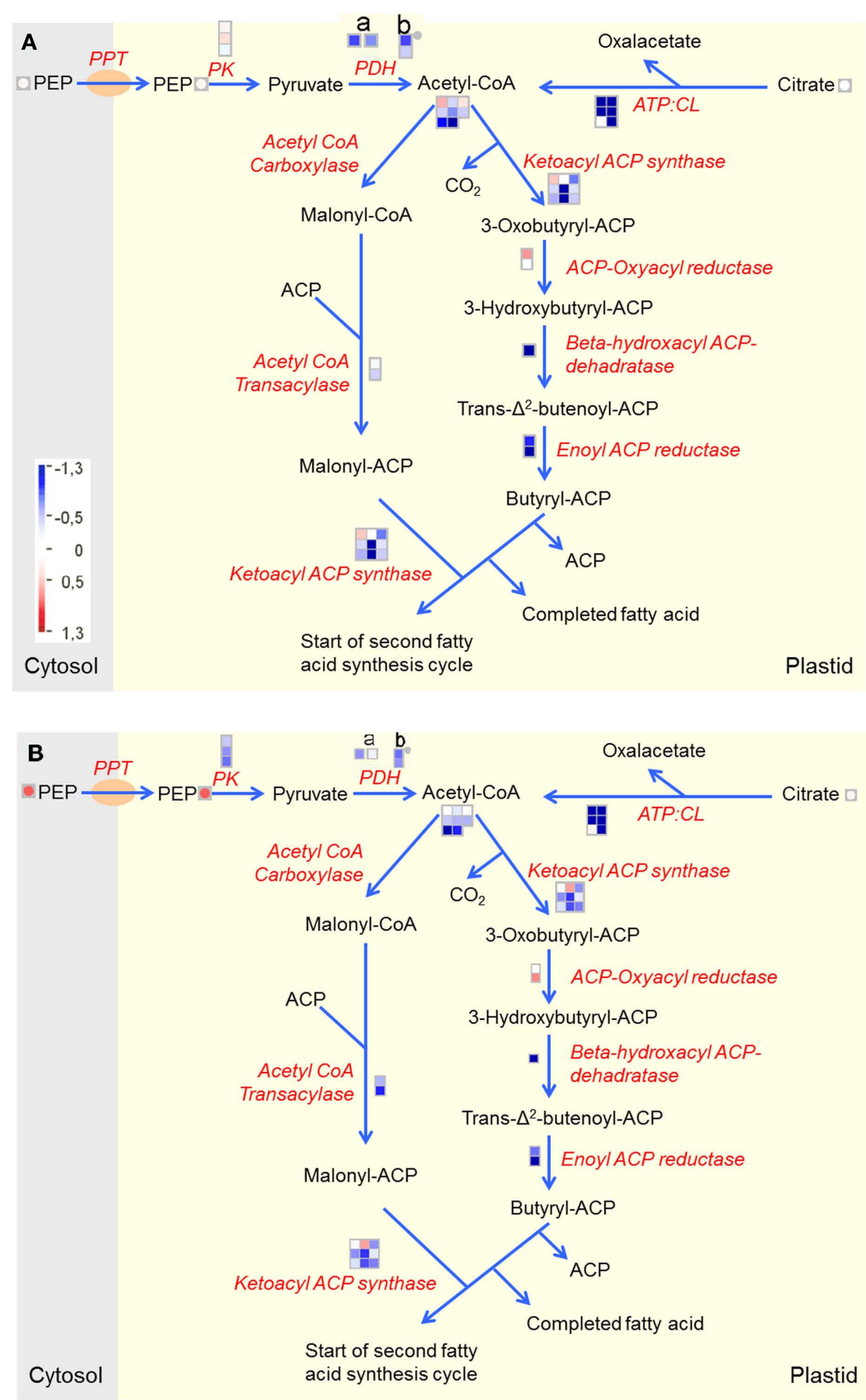

FIGURE 5 | Fatty acid metabolism from imported PEP and citrate in (A) U-IN-1 and (B) U-IN-2. Different subunits of the pyruvate dehydrogenase complex: (a) E2. (b) E3.

in tubers could be explained by the increase in fructose, which is known to be a strong inhibitor of fructokinase (Renz and Stitt, 1993). The product of fructokinase, fructose-6-phosphate (F6P) also serves as an inhibitor of fructokinase activity, a possible reason for slightly reduced expression of fructokinase 2 in U-IN-2. A third isoform present in potato tubers, fructokinase 3 , which in 

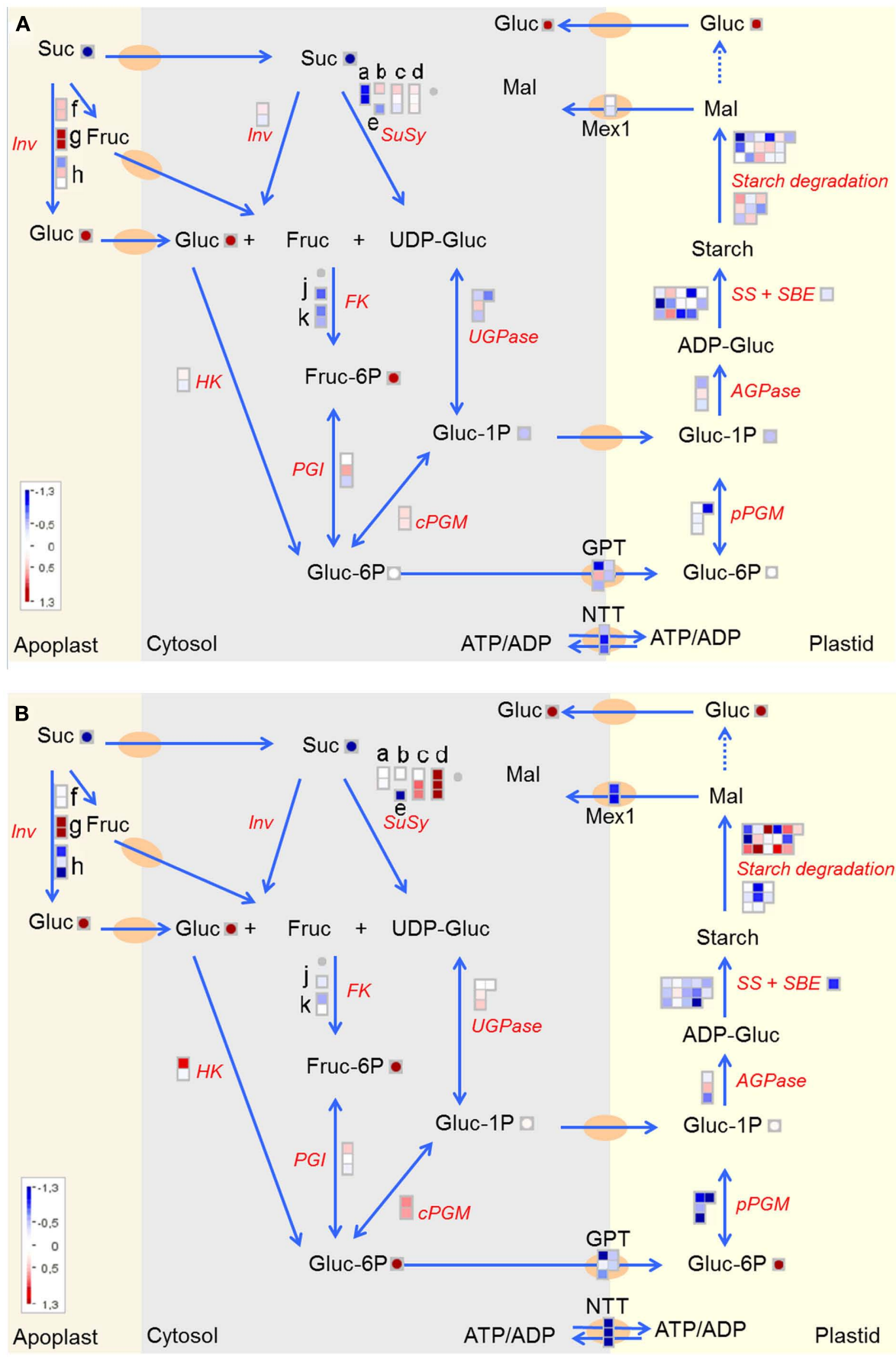

FIGURE 6 | Starch biosynthesis with sucrose as primary substrate in (A) U-IN-1 and (B) U-IN-2. Different subunits of Susy are represented by (a-e) with (a) Susy4. (f-h) Represent the differentially expressed cell wall invertase isoforms. (j) Fructokinase 2. (k) Fructokinase 3. Gene name abbreviations: HK, hexokinase; PGI, phosphoglucose isomerase; SS, starch synthase; SBE, starch branching enzymes; AGPase, ADP-glucose pyrophosphorylase.
Arabidopsis and tomato has been shown to be located in the plastid (German, 2004; Damari-Weissler et al., 2006), was also reduced in both lines. It should be mentioned though that in an earlier study an increase in fructokinase activity in U-IN-2 was found, with no change in U-IN-1 (Hajirezaei et al., 2000), whilst a second study found no significant reduction in fructokinase activity in U-IN-2
(Junker et al., 2004). Not only does this point to the high variability of fructokinase activity in tubers, but also that caution should be exercised when interpreting expression data and its correlation to actual enzyme activity.

Glucose-6-phosphate and glucose-1-phosphate (G1P), although not present in equal amounts, normally shows a constant ratio 
since cytosolic phosphoglucomutase catalyzes a freely reversible reaction (Kossmann and Lloyd, 2000; Periappuram et al., 2000). This was not the case in our study with the ratio of G6P/G1P greatly increased in U-IN-2, indicating that the G6P is not present freely in the cytosol where it can be converted to G1P.

After the production of G6P it must be imported into the plastid, and this occurs via the glucose-6-phosphate transporter, or GPT. In both U-IN-1 and U-IN-2 there was a strong reduction in the expression of the GPT transporter, indicating reduced ability to transport G6P to the plastid. This was despite a significant increase in G6P content in U-IN-2 and could be a result of signaling mechanisms blocking GPT transport under specific conditions (Figure 6). Both, GPT and Susy, positively correlate with storage metabolism, while invertase expression is associated with energy metabolism. Therefore, it was speculated that GPT expression would negatively correlate with invertase expression. To determine whether this is the case, we combined data from this and previous studies performed on growing tubers (Ferreira et al., 2010; Debast et al., 2011) to try and identify a negative correlation between invertase and GPT. Indeed, comparative analysis of the expression patterns showed a strong, inverse correlation between the gene expression pattern for GPT2 and cell wall invertase (Figure 7). This would indicate that under specific "invertase like" conditions, there is a block in GPT mediated G6P transport to the plastid. To get further insight into possible regulatory mechanisms we tested for genes showing either a positive correlation to invertase or GPT expression. Amongst the genes positively correlating to invertase, several transcription factors could be identified (Figure 8). These include auxin response factor 2 (ARF2) and one ABA-response element binding factor (AREB-like protein). In Arabidopsis ARF2 has been suggested not to function within the auxin signaling pathway but to act as negative regulator of cell growth and senescence (Okushima et al., 2005). Amongst others Arabidopsis mutants lacking expression of ARF2 are characterized by a significantly delayed senescence. Hence, elevated expression of ARF2, especially in U-IN-1 tubers, might explain partly the observed induction of senescence-associated genes and possibly acts as negative regulator of GPT. Ectopic expression of SIAREB1, the closest homolog to the identified AREB-like protein, in tomato fruits revealed its involvement in regulating sugar metabolism during fruit development (Bastías et al., 2011). Transgenic fruits expressing SlAREB1 were characterized by increased hexose contents and increased expression of vacuolar invertase. Assuming a similar function in potato tubers, increased expression of the AREB-like protein could be involved in stimulating invertase expression. Interestingly, searching for GPT co-regulated genes did not reveal any

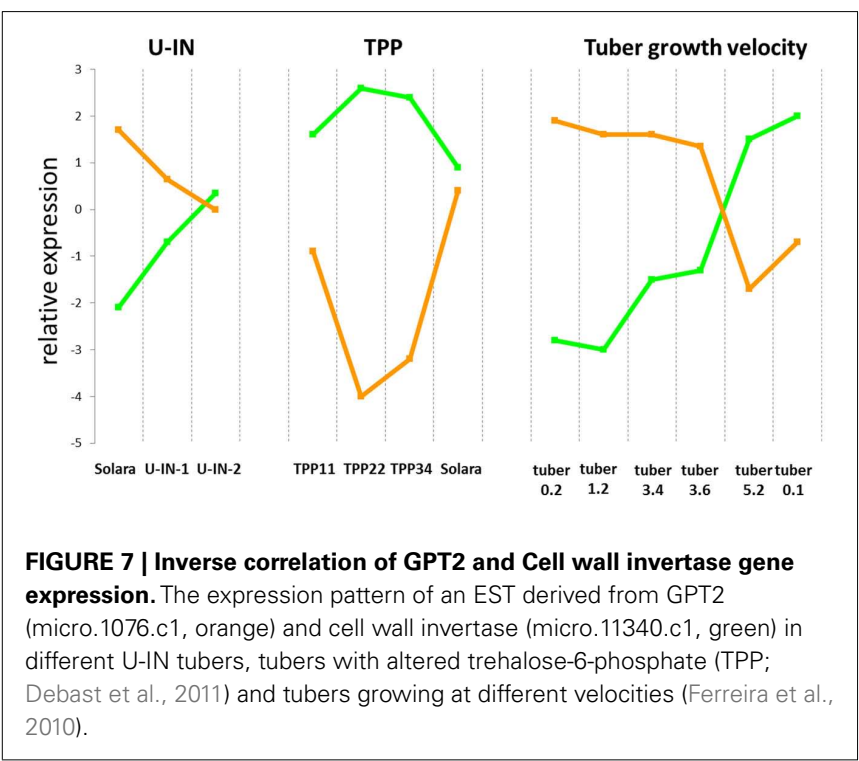

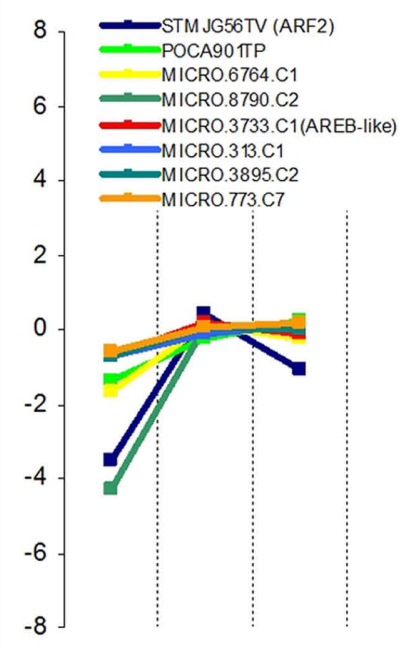

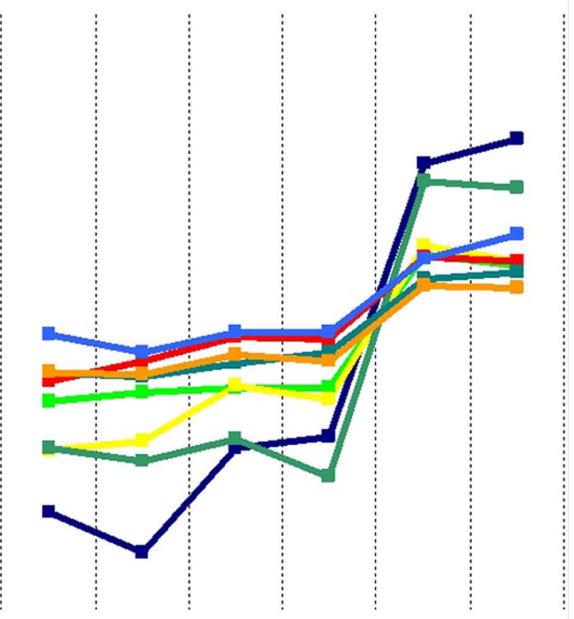

FIGURE 8 | Transcription factors positively correlating to cell wall invertase gene expression. STMJG56TV (ARF2; blue), Micro.8790.c2 (green), POCA901TP (gray), Micro.3733.c1 (AREB-like; purple), Micro.313.c1 (brown) Micro.6764.c1 (yellow), Micro.3895.c1(red), and Micro.773.c7 (orange). 
transcription factor. However a number of starch associated genes showed a strong co-regulation to GPT. These included the plastidic ATP/ADP translocator (NTT; Tjaden et al., 1998), plastidial phosphoglucomutase (pPGM; Tauberger et al., 2000), ADP-glucose pyrophosphorylase (AGPase; Müller-Röber et al., 1992), and granule bound starch synthase (Visser et al., 1991), all required for normal starch biosynthesis. Table S1 in Supplementary Material contains the complete selection of genes co-regulated with either GPT or cell wall invertase.

As mentioned, there is evidence that in potato G1P can also be transported to the plastid, but since the transporter has not yet been identified it is not possible to determine its importance, even at transcriptional level. Transcriptional data on starch degrading enzymes was not clear, but there was a reduction in the expression of MEX1 in U-IN-2, the transporter responsible for the export of the major starch degradation product, maltose, to the cytosol. Earlier studies on U-IN-2 tuber disks had proven inconclusive to whether an increase in flux through starch exists (Trethewey et al., 1999). It does appear from our transcription data that the reason, or at least part of the reason, for reduced starch content in U-IN-2 is that the G6P available cannot be imported to the plastid via GPT, as stated in an earlier study (Farre et al., 2008). Farre et al. (2008) could show by subcellular fractionation experiments that in both genotypes, but especially in U-IN-2, the ratio of cytosolic to plastidial pools of G6P was increased (Figure 6).

\section{REDUCED SUBSTRATE SUPPLY TO THE PLASTID MAY LEAD TO A REDUCED REDOX POTENTIAL AND THEREBY AN ALTERED PLASTID METABOLISM}

Transcriptional and metabolic analysis of the transgenic potato tubers revealed not only changes in carbohydrate metabolism, but also significant changes in metabolites and transcripts of the shikimate and the pentose phosphate pathway, especially in U-IN-2 tubers. This includes an increase in erythrose-4-phosphate, a precursor for shikimate biosynthesis, which pointed toward increased activity of the pentose phosphate pathway, the metabolic route for erythrose-4-phosphate biosynthesis. Indeed, both plastidial isoforms of the rate determining step in the reaction, glucose-6phosphate dehydrogenase $(\mathrm{G} 6 \mathrm{PDH})$, were strongly up-regulated in U-IN-2, whilst there was no change in the expression of the cytosolic form. G6PDH expression and activity, which show strong correlation (Hauschild and von Schaewen, 2003), are activated by a low NADPH/NADP ratio (Wright et al., 1997). The activation of the pentose phosphate pathway would indicate that the plant is compensating for reduced reductive power in the plastid caused by limited G6P supply, producing erythrose-4-phosphate as a product. A reduced NADPH/NADP ratio would also further explain reduced expression of fatty acid biosynthetic genes. Activation of G6PDH would bring the pathway in direct competition with starch biosynthesis regarding the substrate G6P, for which G6PDH has a high affinity under non-reducing conditions (Wenderoth et al., 1997). It should be kept in mind though that an increase in pentose phosphate pathway activity would still represent only a minor flux compared to starch biosynthesis. Moreover, plastidial G6PDH makes up a very small fraction of the total G6PDH activity (von Schaewen et al., 1995), and a strong relative increase in the expression of plastidial G6PDH should be seen in that light. Despite this, the observed activation, at transcriptional level at least, of the pentose phosphate pathway in U-IN-2 does provide evidence of yet another pathway activated at the expense of starch biosynthesis (Figure 9).

The increase in shikimate was surprising in view of the apparent reduced uptake of carbon by the plastid. The pathway, which is unique to plants and micro-organisms, produces chorismate, substrate for the biosynthesis of aromatic amino acids tyrosine, phenylalanine and tryptophan, and is

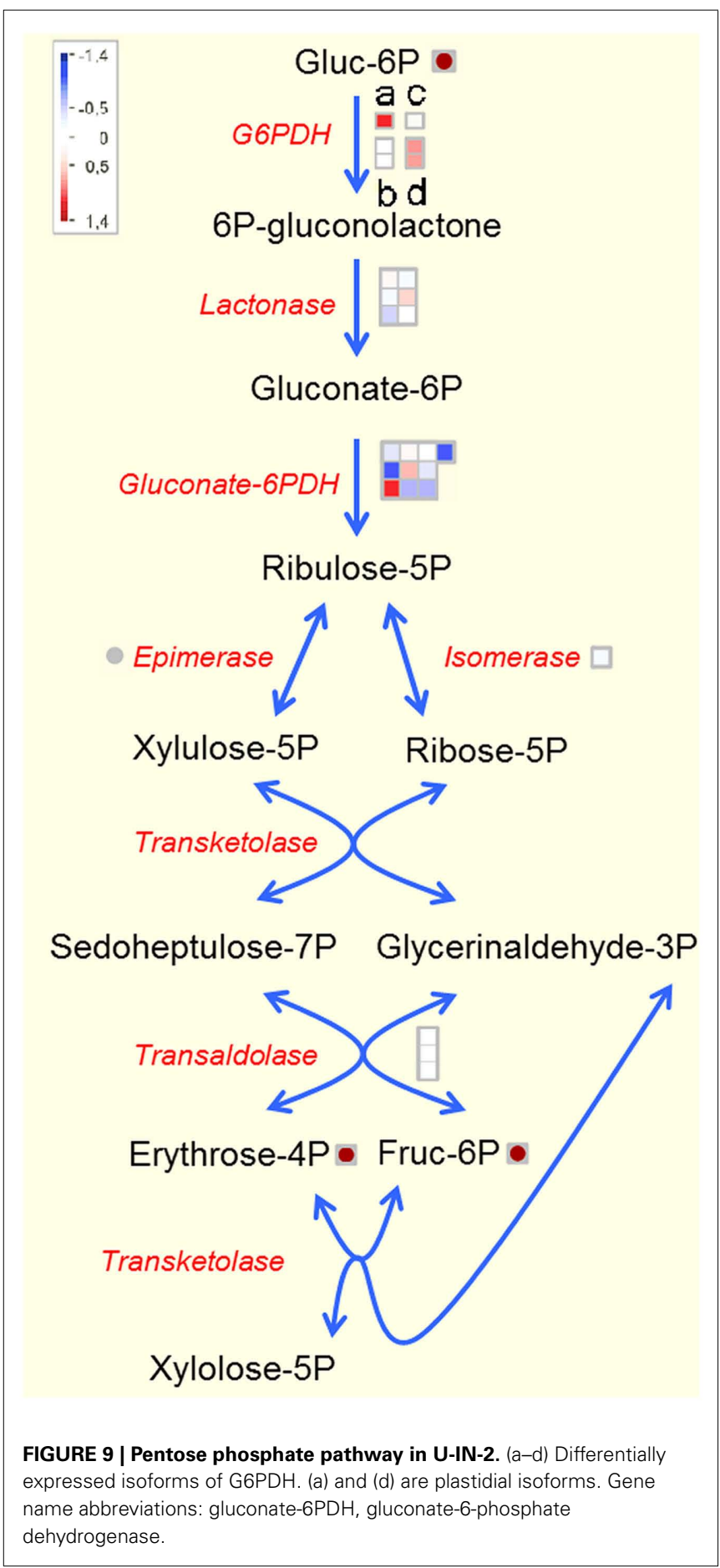


also the precursor for lignin biosynthesis. Yet, despite an increase in shikimate and its precursors PEP, erythrose-4phosphate and 3-deoxy-D-arabino-heptulosonate-7-phosphate (DAHP), there was a general reduction in the expression levels of the enzymes in the pathway, and also of tyrosine, while phenylalanine remained unchanged. There was a strong down-regulation in most of the isoforms of the bifunctional enzyme 3-dehydroquinate dehydrogenase/shikimate-5dehydrogenase (3DHQ DH/shikimate-5-DH). Remarkably, the one isoform which was up-regulated showed high homology to a tobacco gene which was silenced by RNAi (Ding et al., 2007). The silencing led to an unexpected increase in shikimate. Furthermore, phenylalanine lyase (PAL), an enzyme present downstream of phenylalanine toward lignin biosynthesis, was amongst the strongest down-regulated genes in the entire transcriptome analysis.

Although the observed phenotype remains baffling, there are at least three redox dependent steps in the shikimate pathway, 3-dehydroquinate dehydrogenase (3DHQ DH) producing NADPH, shikimate-5-dehydrogenase (shikimate-5-DH) consuming NADPH and chorismate synthase, requiring reduced flavin mononucleotide (FMN) for activity (Macheroux et al., 1999). This would mean that under non-reducing plastidic conditions shikimate would accumulate due to a block in the pathway at this enzymatic step, leading to a depletion of the down-stream metabolites. DAHP synthase, the first committed step in the pathway seems to be under various control mechanisms which differ greatly among species. For instance, it has been shown in Arabidopsis leaves that the enzyme requires $\mathrm{Mn}^{2+}$ and reduced thioredoxin to be active (Entus et al., 2002), which would mean decreased activity under non-reducing conditions. Although regulatory mechanisms acting on DAHP synthase seem to be extensive and not understood, a study by Pinto et al. (1988) provides, to some extent, insight into the observed phenotype in U-IN-2. Their studies on potato cell cultures, treated with sub-lethal doses of glyphosate, showed that inhibition of EPSP synthase can lead to an increase in the activity of DAHP synthase (Pinto et al., 1988). This suggests an inverse regulation of DAHP synthase and EPSP synthase activity, which would lead to shikimate accumulation and reduction of metabolites further down-stream in the pathway. Taken together it seems as though the pathway is subject to multi-level regulation of which redox state is an important contributor and the most likely explanation for the observed changes in U-IN-2 (Figure 10).

\section{SUGAR SIGNALING, POSSIBLY BY WAY OF THE SnRK1 COMPLEX, AND SUBSTRATE CHANNELING APPEAR TO BE THE REASON FOR INCREASED RESPIRATION}

The most striking aspect of U-IN-2 was the increase in respiration, which comes at the expense of starch content, especially in stored tubers (Trethewey et al., 1998; Hajirezaei et al., 2000; Bologa et al., 2003). An increase in respiration should come through the activation of glycolysis and the tricarboxylic acid (TCA) cycle. Expression data, in general, did not reveal a uniform up-regulation of TCA cycle enzymes, although several key metabolic steps were up-regulated. For instance, cytosolic pyruvate kinase and mitochondrial pyruvate dehydrogenase $(\mathrm{mPDH})$ showed strong

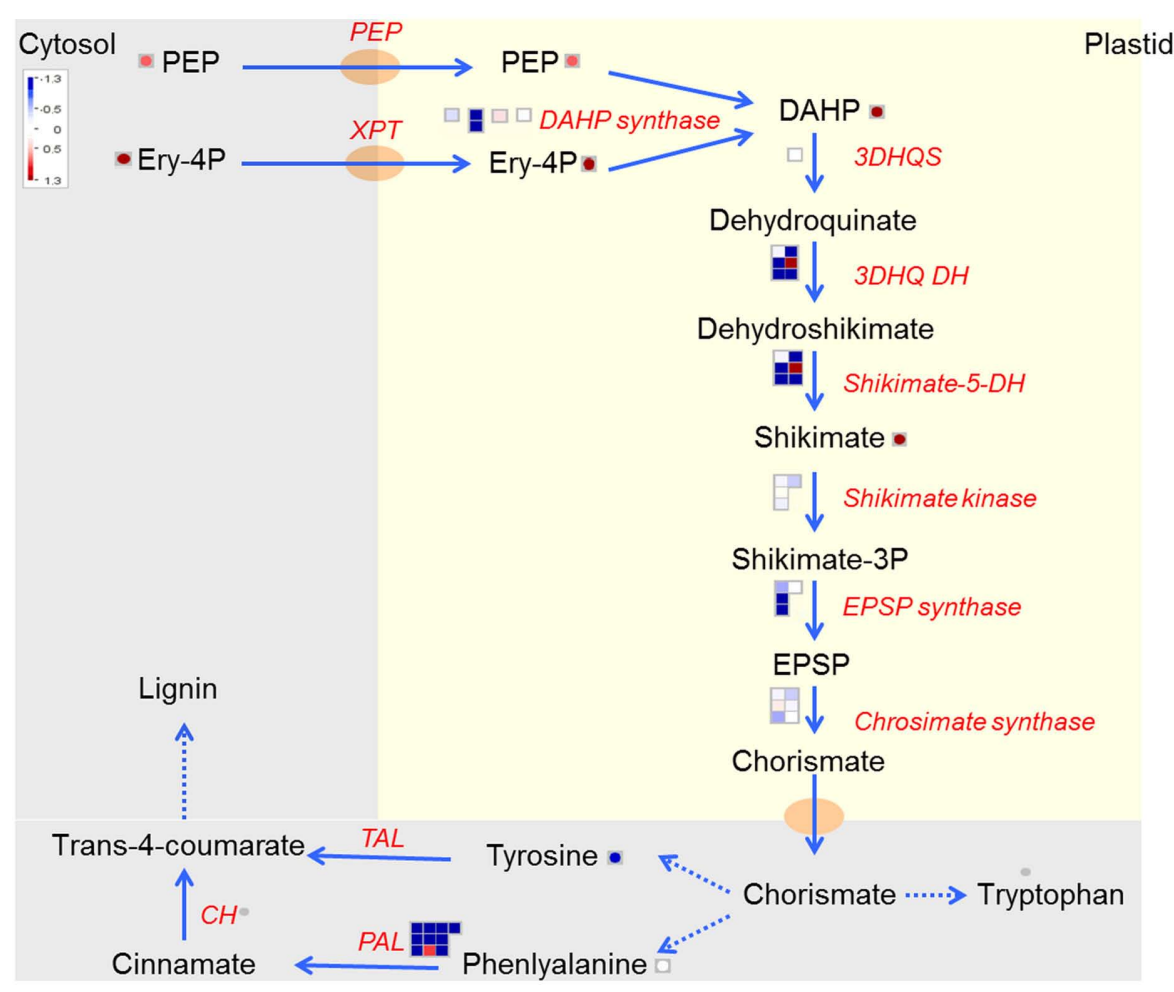

FIGURE 10 | Shikimate biosynthetic pathway producing aromatic amino acids and precursors for lignin in U-IN-2. 
up-regulation, whilst the plastidial isoforms were strongly downregulated, as was seen in the analysis of fatty acid metabolism. All the glycolytic and TCA cycle intermediate metabolites either were unchanged or increased in U-IN-2, with the exception of fumarate which was strongly reduced. As mentioned earlier, there was no significant reduction in the levels of total amino acid contents, which comes as a surprise since most other studies have shown an increase. This might be attributed to differences in growing conditions or sample taking time-points. Importantly, there was an increase in the expression of fructose-2,6-bisphosphatase (F-2,6-BPase). Fructose-2,6-bisphosphate, metabolized by the bifunctional enzyme F-2,6-BPase, is a potent activator of glycolysis by inhibiting FBPase and inducing pyrophosphate: Fru-6-P 1-phosphotransferase (PFP), while phosphofructokinase (PFK) is insensitive to it (Nielsen et al., 2004). Although increased expression of F-2,6-BPase does not necessarily mean increased levels of fructose-2,6-bisphosphate, especially since it is a bifunctional enzyme, this would fit to the observed increase in respiration. Surprisingly, despite the increase in expression of F-2,6-BPase there was a strong increase in the expression of cytosolic fructose1,6-bisphosphatase (FBPase), although the inhibition by fructose2,6-bisphosphate would be at the post-translational level. Phosphorylation of fructose-6-phosphate by PFP rather than PFK is preferred under conditions of low ATP availability. Due to their bulky nature potato tubers have a low internal oxygen concentration. (Bologa et al., 2003) could show that an oxygen gradient from the tuber surface toward the center exists. Moreover, they showed that this gradient was even steeper in U-IN-2. The authors stated that the reason for this is the less energy sufficient sucrose degradation by invertase compared to Susy, as explained earlier. However, this could also be a secondary effect of increased tuber respiration due to other factors. In either case, conditions of hypoxia would lead to the activation of anaerobic respiration, as was shown by Bologa et al. (2003). Although transcription data did not reveal a strong and uniform increase in the expression of marker genes associated with anaerobic respiration, for instance alcohol dehydrogenase $(\mathrm{ADH})$, lactate dehydrogenase (LDH), and glyceraldehyde-3-phosphate dehydrogenase (GA3PDH), there was accumulation of alanine, a marker metabolite for conditions of hypoxia (de Sousa and Sodek, 2003; Miyashita et al., 2007). This would point toward anaerobic respiration taking place at least to some extent, although from our data it does not seem as though this is the predominant form of respiration (Figure 11).

Despite the many studies done on U-IN-2, the reason why there would be an increase in respiration is still unclear. A previous study has suggested that the increase is due to sugar signaling and that the absence of cytosolic sucrose, rather than an increase in

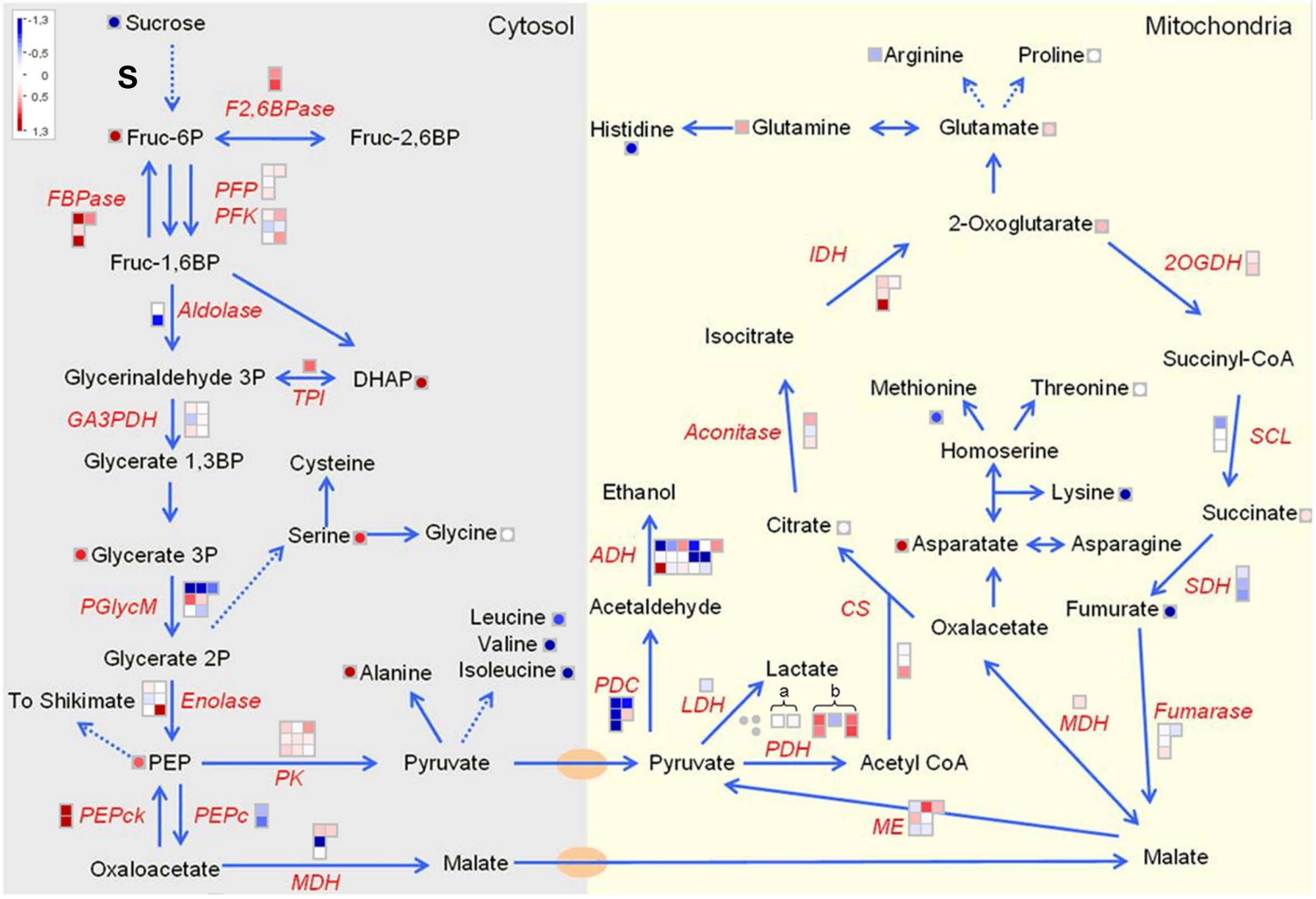

FIGURE 11 | Tricarboxylic acid cycle and respiration in U-IN-2. The E2 (a) and $E 3$ (b) subunits of the mitochondrial pyruvate dehydrogenase complex. Gene name abbreviations: TPI, triose phosphate isomerase; GA3PDH, glyceraldehyde-3-phosphate dehydrogenase; PGlycM, phosphoglycerate mutase; PEPck, PEP carboxylase kinase; PEPc, PEP carboxylase; $\mathrm{MDH}$ malate dehydrogenase; ME, malic enzyme; CS, citrate synthase; IDH, isocitrate dehydrogenase, 2-oxoglutarate dehydrogenase; $\mathrm{SCL}$, succinate $\mathrm{Co} A$ ligase, succinate dehydrogenase. 
glucose, is sensed (Trethewey et al., 2001). This was supported by a study which expressed invertase specifically in the cytosol of companion cells, or reduced cytosolic sucrose by expression of sucrose isomerase. The results indicated that a depletion of cytosolic sucrose, and not increased hexose metabolism, lead to the increase in respiration (Hajirezaei et al., 2003). This would indicate that it is highly likely that sugar signaling is primarily responsible for increased respiration, although the mechanisms behind it still need to be resolved. Inspired by the fact that elevated respiration is characteristic for U-IN-2 but not U-IN-1 tubers we were interested to identify possible regulators highly expressed in U-IN-2 but not in U-IN-1. This analysis revealed a significant list of differentially expressed genes but the gene showing the strongest induction in U-IN-2 tubers encodes for a catalytic trehalose phosphatase (Table S2 in Supplementary Material). Assuming that increased expression of the gene would lead to a higher enzyme activity, a decreased accumulation of trehalose6-phosphate in U-IN-2 tubers would be expected. This would result in activation of SnRK1 and hence most likely enhanced catabolic metabolism including respiration. Elevated respiration of trehalose phosphatase overexpressing transgenic potato tubers has recently been published (Debast et al., 2011), supporting our hypothesis. Since this trehalose phosphatase is not induced in $\mathrm{U}$ IN-1 tubers, it could explain the observed difference between both genotypes with respect to respiratory activities. Attempts to measure T6P accumulation in both genotypes were handicapped due to the very low T6P level, which was below detection limit for both genotypes.

Recent studies in Arabidopsis and potato have shown that there is a correlation between increased respiration and glycolytic enzyme association with the mitochondrial membrane, suggesting the channeling of carbon toward the mitochondria occurs under specific conditions. Moreover, it could be show that this increased association of glycolytic enzymes to the mitochondrial membrane also exists in U-IN-2 when compared to control tubers (Graham et al., 2007). The entry point to this proposed channel is currently not well understood, especially in plants. In mammalian systems, however, it has been proposed that different hexokinase isoforms are important to channel G6P into different metabolic routes, and that mitochondrial-bound hexokinase would channel the carbon toward mitochondria and glycolysis (Wilson, 2003). Although direct evidence is still lacking, we suggest a similar mechanism where hexokinase 5, in response to specific sugar signals, channels glucose through phosphorylation toward glycolysis and respiration (Figure 12). Further evidence for this is the imbalance in the ratio between G6P and G1P, indicating that the G6P is caught in the complex and not accessible to CPGM. We propose that a possible means to overcome this problem would be to introduce a second complex of proteins involved in sucrose cleavage and the production of G6P, anchored to the plastid membrane via GPT. Future work will be aimed at testing this hypothesis.

\section{CONCLUSION}

Our data show that accumulation of apoplasmic and symplasmic hexoses lead to different metabolic signals activating different metabolic and physiological processes. In both genotypes there was a reduction of cell wall biosynthetic gene expression, explained by reduced production of UDP-glucose. We provide evidence for substrate channeling which shifts metabolic flux toward respiration and speculate on the involvement of the SnRK1 complex. Furthermore, a strict negative correlation between GPT and cell wall invertase, possibly mediated by transcription factors, suggest that under "invertase like" conditions plastid transporters are down-regulated, leading to insufficient substrate supply. Reduced supply, resulting in a changed redox state, could explain the indirect effects of reduced fatty acid biosynthetic gene expression and the accumulation of shikimate.

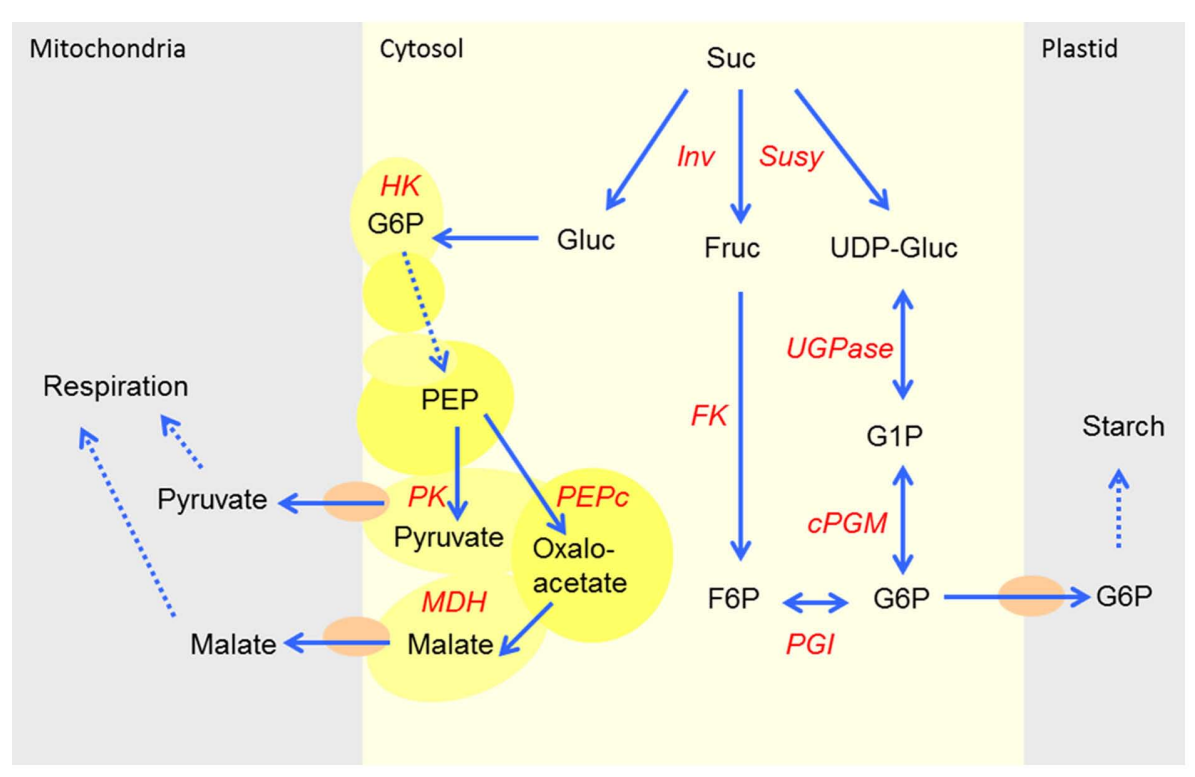

FIGURE 12 | Proposed channeling of glucose toward the glycolysis and respiration with hexokinase as the entry point. 


\section{MATERIALS AND METHODS PLANTS AND GROWTH CONDITIONS}

Solanum tuberosum L. cv. Desiree and transgenic lines U-IN-1-33 and U-IN-2-17 (Sonnewald et al., 1997) were maintained in tissue culture on MS medium (Murashige and Skoog, 1962) containing $2 \%(\mathrm{w} / \mathrm{v})$ sucrose with a 16 -h light and $8 \mathrm{~h}$ dark regime. After transfer to the greenhouse the selected lines were cultivated in individual $4 \mathrm{~L}$ pots at $50 \%$ humidity with $16 \mathrm{~h}$ of supplemental light (150 $\mu$ mol quanta $\mathrm{m}^{-2} \mathrm{~s}^{-1}$ ) and $8 \mathrm{~h}$ of darkness. The temperature regime followed the light/dark cycle with 21 and $18^{\circ} \mathrm{C}$

\section{SAMPLING AND RNA EXTRACTION}

After 8 weeks potato tubers were harvested and samples taken using a cork borer. Only material from the center of the tuber was kept and immediately frozen in liquid nitrogen. Potato tubers from each individual plant were pooled and the different plants were treated as biological replicates. After sample taking material was stored at $-80^{\circ} \mathrm{C}$. RNA was extracted as described previously (Logemann et al., 1987). Four replicates for Desiree and three for each of the transgenic lines were used for microarray hybridization.

\section{MICROARRAY HYBRIDIZATION}

Microarray hybridizations were performed as described previously (Ferreira et al., 2010).

\section{DATA EXTRACTION AND ANALYSIS}

For data analysis, text files generated by feature extraction software were imported into GeneSpring ${ }^{\mathrm{TM}}$ (version 11.0). Three normalization steps were applied: (1) data transformation: measurements less than 1.0 were set to 1.0 ; (2) per chip: normalization to the 75th percentile; (3) per gene: the signal of each feature was normalized to the median of the control samples. For MapMan analysis an ANOVA test was performed to select the features which were significantly altered from the control $(p \leq 0.05$, variance assumed as equal). For both PCA analysis and pathway specific analysis, the fold change, relative to the control, was calculated and used for subsequent analysis. Furthermore, to make the data compatible for MapMan analysis, the $\log 2$ value was calculated. PCA analysis was performed using MarkerView ${ }^{\mathrm{TM}}$ software (version 1.1.0.7) and the Pareto clustering algorithm was used. For the functional assignment a volcano plot $(p \leq 0.05$, variance assumed as equal; features changed more than twofold changed relative to the control) was performed and selected features were divided into up- and down-regulated groups. The percentage of features from a specific functional group was expressed relative to the percentage of features from that group on the entire chip. Functional groupings were used as described previously (Hartmann et al., 2011). For analysis with MapMan software the POCI mapping file was downloaded from the MapMan website

\section{REFERENCES}

Amor, Y., Haigler, C. H., Johnson, S., Wainscott, M., and Delmer, D. P. (1995). A membrane-associated form of sucrose synthase and its potential role in synthesis of cellulose and callose in plants. Proc. Natl. Acad. Sci. U.S.A. 92, 9353-9357.
Avigad, C. (1982). "Encyclopedia of plant physiology," in Storage Carbohydrates in Vascular Plants, 53-73.

Barratt, D. H. P., Derbyshire, P., Findlay, K., Pike, M., Wellner, N., Lunn, J., Feil, R., Simpson, C., Maule, A. J., and Smith, A. M. (2009). Normal growth of Arabidopsis requires cytosolic

(https://www.gabipd.org/projects/MapMan/). In certain cases the mappings were changed to allow the separation of genes coding for proteins expressed in different subcellular compartments, and also to separate features derived from different genes. The mapping file with changes can be found in the Table S3 in Supplementary Material. The entire microarray dataset has been deposited on ArrayExpress (Accession number E-MEXP-3338).

To identify features expressed in U-IN-2, but not in U-IN1 , a volcano plot $(p \leq 0.05$, variance assumed as equal; features changed more than twofold changed in U-IN-2 relative to the control) was used to select differentially expressed features and these were exported to Microsoft Excel. Features which were also regulated in U-IN-1 (relative $\log 2$ expression value more than 0.5 , or less than -0.5) were excluded (Table S2 in Supplementary Material).

For the selection of features co-expressed with either GPT2 (micro.1076.c1) or cell wall invertase (micro.11340.c1), selected datasets were imported into GeneSpring and a new experiment was created. Three normalization steps were applied: (1) data transformation: measurements less than 1.0 were set to 1.0 ; (2) per chip: normalization to the 75th percentile; (3) per gene: the signal of each feature was normalized to the median of its value across the dataset. Features with a correlation coefficient (Pearson correlation, $t$-test unpaired) of $>0.95$ were selected for micro.1076.c1 and micro.11340.c1 respectively (Table S1 in Supplemental Material).

\section{METABOLITE EXTRACTION, MEASUREMENT, AND ANALYSIS}

Metabolite extraction and measurement of organic acids, phosphorylated intermediates, amino acids, carotenoids (Horst et al., 2010), major soluble sugars, and starch (Müller-Röber et al., 1992) was performed as described previously. For both PCA analysis and pathway specific analysis, the fold change, relative to the control, was calculated and used for subsequent analysis. Furthermore, to make the data compatible for MapMan analysis, the $\log 2$ value was calculated.

\section{ACKNOWLEDGMENTS}

We would like to thank Dr. Joerg Hofmann for HPLC analysis. Stephen Reid, Christiane Boernke, and Alfred Schmiedl provided excellent technical assistance, and Christine Hoesl took care of the plants in the greenhouse. The work was funded by the German ministry for education and research $(\mathrm{BMBF})$ in the frame of the GABI-FUTURE program.

\section{SUPPLEMENTARY MATERIAL}

The Supplementary Material for this article can be found online at http://www.frontiersin.org/plant_physiology/10.3389/fpls.2012. 00023/abstract

invertase but not sucrose synthase. Proc. Natl. Acad. Sci. U.S.A .106, 13124-13129.

Bastías, A., López-Climent, M., Valcárcel, M., Rosello, S., GómezCadenas, A., and Casaretto, J. A (2011). Modulation of organic acids and sugar content in tomato fruits by an abscisic acid-regulated transcription factor. Physiol. Plant. 141, 215-226.

Bologa, K. L., Fernie, A. R., Leisse, A., Loureiro, M. E., and Geigenberger, P. (2003). A bypass of sucrose synthase leads to low internal oxygen and impaired metabolic performance in 
growing potato tubers. Plant Physiol. 132, 2058-2072.

Contento, A. L., Kim, S.-J., and Bassham, D. C. (2004). Transcriptome profiling of the response of Arabidopsis suspension culture cell to Suc starvation. Plant Physiol. 135, 2330-2347.

Damari-Weissler, H., Kandel-Kfir, M., Gidoni, D., Mett, A., Belausov, E., and Granot, D. (2006). Evidence for intracellular spatial separation of hexokinases and fructokinases in tomato plants. Planta 224, 1495-1502.

de Sousa, C. A. F., and Sodek, L. (2003). Alanine metabolism and alanine aminotransferase activity in soybean (Glycine max) during hypoxia of the root system and subsequent return to normoxia. Environ. Exp. Bot. 50, $1-8$.

Debast, S., Nunes-Nesi, A., Hajirezaei, M.-R., Hofmann, J., Sonnewald, U., Fernie, A. R., and Börnke, F. (2011). Altering trehalose-6-phosphate content in transgenic potato tubers affects tuber growth and alters responsiveness to hormones during sprouting. Plant Physiol. 156, 1-19.

Ding, L., Hofius, D., Hajirezaei, M.R., Fernie, A. R., Börnke, F., and Sonnewald, U. (2007). Functional analysis of the essential bifunctional tobacco enzyme 3-dehydroquinate dehydratase/shikimate dehydrogenase in transgenic tobacco plants. $J$. Exp. Bot. 58, 2053-2067.

Entus, R., Poling, M., and Herrmann, K. M. (2002). Redox regulation of Arabidopsis 3-deoxy-D-arabinoheptulosonate-7-phosphate synthase 1 . Society 129, 1866-1871.

Farre, E. M., Fernie, A. R., and Willmitzer, L. (2008). Analysis of subcellular metabolite levels of potato tubers (Solanum tuberosum) displaying alterations in cellular or extracellular sucrose metabolism. Metabolomics 4, 161-170.

Ferreira, S. J., Senning, M., Sonnewald, S., Kessling, P.-M., Goldstein, R., and Sonnewald, U. (2010). Comparative transcriptome analysis coupled to X-ray CT reveals sucrose supply and growth velocity as major determinants of potato tuber starch biosynthesis. BMC Genomics 11, 93. doi:10.1186/1471-2164-11-93

Fettke, J., Albrecht, T., Hejazi, M., Mahlow, S., Nakamura, Y., and Steup, M. (2010). Glucose 1phosphate is efficiently taken up by potato (Solanum tuberosum) tuber parenchyma cells and converted to reserve starch granules. New Phytol. 185, 663-675.

Flügge, U.-I., Häusler, R. E., Ludewig, F., and Gierth, M. (2011). The role of transporters in supplying energy to plant plastids. J. Exp. Bot. 62, 2381-2392.

Fu, H., and Park, W. D. (1995). Sink- and vascular-associated sucrose synthase functions are encoded by different gene classes in potato. Plant Cell 7, 1369-1385.

German, M. (2004). Cloning, expression and characterization of Lefrk3, the fourth tomato (Lycopersicon esculentum mill.) gene encoding fructokinase. Plant Sci. 166, 285-291.

Giese, J.-O. (2004). Molekulare Und Biochemiese Charakterisierung De Hexokinase-Genfamilie Von Nicotiana Tabacum. Doctoral thesis, Halle.

Graham, J. W. A., Williams, T. C. R., Morgan, M., Fernie, A. R., Ratcliffe, R. G., and Sweetlove, L. J. (2007). Glycolytic enzymes associate dynamically with mitochondria in response to respiratory demand and support substrate channeling. Plant Cell 19, 3723-3738.

Hajirezaei, M. R., Takahata, Y., Trethewey, R. N., Willmitzer, L., and Sonnewald, U. (2000). Impact of elevated cytosolic and apoplastic invertase activity on carbon metabolism during potato tuber development. J. Exp. Bot. 51, 439-445.

Hajirezaei, M.-R, Börnke, F., Peisker, M., Takahata, Y., Lerchl, J., Kirakosyan, A., and Sonnewald, U. (2003). Decreased sucrose content triggers starch breakdown and respiration in stored potato tubers (Solanum tuberosum). J. Exp. Bot. 54, 477-488.

Hartmann, A., Senning, M., Hedden, P., Sonnewald, U., and Sonnewald, S. (2011). Reactivation of meristem activity and sprout growth in potato tubers require both cytokinin and gibberellin. Plant Physiol. 155, 776-796.

Hauschild, R., and von Schaewen, A. (2003). Differential regulation of glucose-6-phosphate dehydrogenase isoenzyme activities in potato. Plant Physiol. 133, 47-62.

Heineke, D., Sonnewald, U., Büssis, D., Günter, G., Leidreiter, K., Wilke, I., Raschke, K., Willmitzer, L., and Heldt, H. W. (1992). Apoplastic expression of yeast-derived invertase in potato: effects on photosynthesis, leaf solute composition, water relations, and tuber composition. Plant Physiol. 100, 301-308.

Horst, R. J., Doehlemann, G., Wahl, R., Hofmann, J., Schmiedl, A., Kahmann, R., Kaemper, J., Sonnewald, U., and Voll, L. M. (2010). Ustilago maydis infection strongly alters organic nitrogen allocation in maize and stimulates productivity of systemic source leaves. Plant Physiol. 152, 293-308.

Junker, B. H., Wuttke, R., Tiessen, A., Geigenberger, P., Sonnewald, U., Willmitzer, L., and Fernie, A. R. (2004). Temporally regulated expression of a yeast invertase in potato tubers allows dissection of the complex metabolic phenotype obtained following its constitutive expression. Plant Mol. Biol. 56, 91-110.

Keech, O., Pesquet, E., Gutierrez, L., Ahad, A., Bellini, C., Smith, S. M., and Gardeström, P. (2010). Leaf senescence is accompanied by an early disruption of the microtubule network in Arabidopsis. Plant Physiol. 154, 1710-1720.

Kloosterman, B., De Koeyer, D., Griffiths, R., Flinn, B., Steuernagel, B. Scholz, U., Sonnewald, S., Sonnewald, U., Bryan, G. J., Prat, S., Bánfalvi, Z., Hammond, J. P., Geigenberger, P., Nielsen, K. L., Visser, R. G. F., and Bachem, C. W. B. (2008). Genes driving potato tuber initiation and growth: identification based on transcriptional changes using the POCI array. Funct. Integr. Genomics 8, 329-340.

Kocal, N., Sonnewald, U., and Sonnewald, S. (2008). Cell wall-bound invertase limits sucrose export and is involved in symptom development and inhibition of photosynthesis during compatible interaction between tomato and Xanthomonas campestris pv Vesicatoria. Plant Physiol. 148, 1523-1536.

Kohlhaw, G. B., and Tan-Wilson, A. (1977). Carnitine acetyltransferase: candidate for the transfer of acetyl groups through the mitochondrial membrane of yeast. J. Bacteriol. 129. 1159-1161.

Kossmann, J., and Lloyd, J. (2000). Understanding and influencing starch biochemistry. Crit. Rev. Biochem. Mol. Biol. 35, 141-196.

Kötting, O., Kossmann, J., Zeeman, S. C., and Lloyd, J. R. (2010). Regulation of starch metabolism: the age of enlightenment? Curr. Opin. Plant Biol. 13, 321-329.

Loewus, F. A., and Loewus, M. W. (1980). Myo-inositol-1-phosphatase from the pollen of Lilium longiflorum Thunb. Plant Physiol. 70, 765-770.

Loewus, M. W., Loewus, F. A., Brillinger, G. U., Otsuka, H., and Floss, H. G. (1980). Stereochemistry of the myo-inositol-1-phosphate synthase reaction. J. Biol. Chem. 255, 11710-11712.
Logemann, J., Schell, J., and Willmutzer, L. (1987). Improved method for the isolation of RNA from plant tissues. Anal. Biochem. 20, 16-20.

Lytovchenko, A., Eickmeier Pons, C., Osorio, S., Szecowka, M., Lehmberg, K., Arrivault, S., Tohge, T., Pineda B., Anton, M. T., Hedtke, B., Lu, Y., Fisahn, J., Bock, R., Stitt, M. Grimm, B., Granell, A., and Fernie, A. R. (2011). Tomato fruit photosynthesis is seemingly unimportant in primary metabolism and ripening but plays a considerable role in seed development. Plant Physiol. 157, 1650-1663.

Macheroux, P., Schmid, J., Amrhein, N., and Schaller, A. (1999). A unique reaction in a common pathway: mechanism and function of chorismate synthase in the shikimate pathway. Planta 207, 325-334.

Miyashita, Y., Dolferus, R., Ismond, K. P., and Good, A. G. (2007). Alanine aminotransferase catalyses the breakdown of alanine after hypoxia in Arabidopsis thaliana. Plant J. 49, 1108-1121.

Müller-Röber, B., Sonnewald, U., and Willmitzer, L. (1992). Inhibition of the ADP-glucose pyrophosphorylase in transgenic potatoes leads to sugarstoring tubers and influences tuber formation and expression of tuber storage protein genes. EMBO J. 11, 1229-1238.

Murashige, T., and Skoog, F. (1962). A revised medium for rapid growth and bio assays with tobacco tissue cultures. Physiol. Plant. 15, 473-497.

Nielsen, T. H., Rung, J. H., and Villadsen, D. (2004). Fructose-2,6bisphosphate: a traffic signal in plant metabolism. Trends Plant Sci. 9, 556-563.

Okushima, Y., Mitina, I., Quach, H. L., and Theologis, A. (2005). Auxin response factor 2 (Arf2): a pleiotropic developmental regulator. Plant J. 43, 29-46.

Patel, T. B., and Clark, J. B. (1980). Lipogenesis in the brain of suckling rats. Studies on the mechanism of mitochondrial-cytosolic carbon transfer. Biochem. J. 188, 163-168.

Periappuram, C., Steinhauer, L., Barton, D. L., Taylor, D. C., Chatson, B., and Zou, J. (2000). The plastidic phosphoglucomutase from Arabidopsis. A reversible enzyme reaction with an important role in metabolic control. Plant Physiol. 122, 1193-1199.

Pinto, J. E., Dyer, W. E., Weller, S. C., and Herrmann, K. M. (1988). Glyphosate induces 3-deoxy-Darabino-heptulosonate 7-phosphate synthase in potato (Solanum tuberosum L.) cells grown in 
suspension culture. Plant Physiol. 87, 891-893.

Rangasamy, D., and Ratledge, C. (2000). Compartmentation of ATP:citrate lyase in plants. Plant Physiol. 122, 1225-1230.

Renz, A., and Stitt, M. (1993). Substrate specificity and product inhibition of different forms of fructokinases and hexokinases in developing potato tubers. Planta 190, 166-175

Rocha-Sosa, M., Sonnewald, U., Frommer, W. B., Stratmann, M., Schell, J., and Willmitzer, L. (1989). Both the developmental and metabolic signals activate the promoter of a class I gene. EMBO J. 8, 23-29.

Roitsch, T., Bittner, M., and Godt, D. E. (1995). Induction of apoplastic invertase of Chenopodium rubrum by $\mathrm{D}$-glucose and a glucose analog and tissue-specific expression suggest a role in sink-source regulation. Plant Physiol. 108, 285-294.

Roitsch, T., and González, M.-C. (2004). Function and regulation of plant invertases: sweet sensations. Trends Plant Sci. 9, 606-613.

Smeekens, S., Ma, J., Hanson, J., and Rolland, F. (2010). Sugar signals and molecular networks controlling plant growth. Curr. Opin. Plant Biol. 13, 274-279.

Sonnewald, U., Hajirezaei, M.-R., Kossmann, J., Heyer, A., Trethewey, R. N., and Willmitzer, L. (1997). Increased potato tuber size resulting from the apoplastic expression of a yeast invertase. Nat. Biotechnol. 15, 794-797.

Tauberger, E., Fernie, A. R., Emmermann, M., Renz, A., Kossmann, J., Willmitzer, L., and Trethewey, R. N. (2000). Antisense inhibition of plastidial phosphoglucomutase provides compelling evidence that potato tuber amyloplasts import carbon from the cytosol in the form of glucose-6-phosphate. Plant J. 23, 43-53.

Thimm, O., Bläsing, O., Gibon, Y., Nagel, A., Meyer, S., Krüger, P., Selbig, J., Müller, L. A., Rhee, S. Y., and Stitt, M. (2004). Mapman: a user-driven tool to display genomics data sets onto diagrams of metabolic pathways and other biological processes. Plant J. 37, 914-939.

Tjaden, J., Mohlmann, T., Kampfenkel, K., and Neuhaus, G. H. A. E. (1998). Altered plastidic ATP/ADPtransporter activity influences potato (Solanum tuberosum L.) tuber morphology, yield and composition of tuber starch. Plant J. 16, 531-540.

Trethewey, R. N., Fernie, A. R., Bachmann, A., Fleischer-Notter, H., Geigenberger, P., and Willmitzer, L. (2001). Expression of a bacterial sucrose phosphorylase in potato tubers results in a glucoseindependent induction of glycolysis. Plant Cell Environ. 24, 357-365.

Trethewey, R. N., Geigenberger, P., Hennig, A., Fleischer-Notter, H., MullerRober, B., and Willmitzer, L. (1999). Induction of the activity of glycolytic enzymes correlates with enhanced hydrolysis of sucrose in the cytosol of transgenic potato tubers. Plant Cell Environ. 22, 71-79.

Trethewey, R. N., Geigenberger, P., Riedel, K., Hajirezaei, M. R., Sonnewald, U., Stitt, M., Riesmeier, J. W., and Willmitzer, L. (1998). Combined expression of glucokinase and invertase in potato tubers leads to a dramatic reduction in starch accumulation and a stimulation of glycolysis. Plant J. 15, 109-118.

Urbanczyk-Wochniak, E., Luedemann, A., Kopka, J., Selbig, J., RoessnerTunali, U., Willmitzer, L., Fernie, A. R. (2003). Parallel analysis of transcript and metabolic profiles: a new approach in systems biology. $E M B O$ Rep. 4. 989-993.

Vargas, W. A., Pontis, H. G., and Salerno, G. L. (2008). New insights on sucrose metabolism: evidence for an active $\mathrm{N}$-Inv in chloroplasts uncovers a novel component of the intracellular carbon trafficking. Planta 227, 795-807.

Viola, R., Roberts, A. G., Haupt, S., Gazzani, S., Hancock, R. D., Marmiroli, N., Machray, G. C., and Oparka, K. J. (2001). Tuberization in potato involves a switch from apoplastic to symplastic phloem unloading. Plant Cell 13, 385-398.

Visser, R. G., Somhorst, I., Kuipers, G. J., Ruys, N. J., Feenstra, W. J., and Jacobsen, E. (1991). Inhibition of the expression of the gene for granulebound starch synthase in potato by antisense constructs. Mol. Gen. Genet. 225, 289-296.

von Schaewen, A., Langenkämper, G., Graeve, K., Wenderoth, I., and Scheibe, R. (1995). Molecular characterization of the plastidic glucose6-phosphate dehydrogenase from potato in comparison to its cytosolic counterpart. Plant Physiol. 109, 1327-1335.

Wenderoth, I., Scheibe, R., and von Schaewen, A. (1997). Identification of the cysteine residues involved in redox modification of plant plastidic glucose-6-phosphate dehydrogenase. J. Biol. Chem. 272, 26985-26990.

Wilson, J. E. (2003). Isozymes of mammalian hexokinase: structure, subcellular localization and metabolic function. J. Exp. Biol. 206 2049-2057.

Wingler, A. (1998). Regulation of leaf senescence by cytokinin, sugars, and light. Effects on NADH-dependent hydroxypyruvate reductase. Plant Physiol. 116, 329-335.

Wingler, A., and Roitsch, T. (2008) Metabolic regulation of leaf senescence: interactions of sugar signalling with biotic and abiotic stress responses. Plant Boil. (Stuttg.) 10 50-62.

Wright, D. P., Huppe, H. C., and Turpin, D. H. (1997). In vivo and in vitro studies of glucose-6phosphate dehydrogenase from barley root plastids in relation to reductant supply for No, assimilation. Plant Physiol. 114, 1413-1419.

Xu, X., Pan, S., Cheng, S., Zhang, B., $\mathrm{Mu}$, D., Ni, P., Zhang, G., Yang, S., Li, R., Wang, J., Orjeda, G., Guzman, F., Torres, M., Lozano, R., Ponce, O., Martinez, D., De la Cruz, G., Chakrabarti, S. K., Patil, V. U., Skryabin, K. G., Kuznetsov, B. B. Ravin, N. V., Kolganova, T. V., Beletsky, A. V., Mardanov, A. V., Di Genova, A., Bolser, D. M., Martin, D., Ma Li, G., Yang, Y., Kuang, H., Hu, Q., Xiong, X., Bishop, G. J., Sagredo, B., Mejía, N., Zagorski, W., Gromadka, R., Gawor, J., Szczesny, P., Huang, S., Zhang, Z., Liang, C., He, J., Li, Y., He, Y., Xu, J., Zhang, Y., Xie, B., Du, Y., Qu, D., Bonierbale, M., Ghislain, M. Del Rosario Herrera, M., Giuliano, G., Pietrella, M., Perrotta, G., Facella, P., O’Brien, K., Feingold, S. E., Barreiro, L. E., Massa, G. A., Diambra, L., Whitty, B. R., Vaillancourt, B. Lin, H., Massa, A. N., Geoffroy, M., Lundback, S., Dellapenna, D., Robin Buell, C., Sharma, S. K., Marshall, D. F., Waugh, R., Bryan, G. J., Destefanis, M., Nagy, I., Milbourne, D., Thomson, S. J., Fiers, M., Jacobs, J. M. E., Nielsen, K. L., Sønderkær, M.,
Iovene, M., Torres, G. A., Jiang, J., Veilleux, R. E., Bachem, C. W. B., de Boer, J., Borm, T., Kloosterman, B., van Eck, H., Datema, E., Te Lintel Hekkert, B., Goverse, A., van Ham, R. C. H. J., and Visser, R. G. F. (2011). Genome sequence and analysis of the tuber cop potato. Nature 475, 189-195.

Zhang, L., Häusler, R. E., Greiten, C., Hajirezaei, M.-R., Haferkamp, I., Neuhaus, H. E., Flügge, U.I., and Ludewig, F. (2008). Overriding the co-limiting import of carbon and energy into tuber amyloplasts increases the starch content and yield of transgenic potato plants. Plant Biotechnol. J. 6, 453-464.

Zrenner, R., Krause, K. P., Apel, P., and Sonnewald, U. (1996). Reduction of the cytosolic fructose-1,6bisphosphatase in transgenic potato plants limits photosynthetic sucrose biosynthesis with no impact on plant growth and tuber yield. Plant J. 9, 671-681.

Zrenner, R., Salanoubat, M., Willmitzer, L., and Sonnewald, U. (1995). Evidence of the crucial role of sucrose synthase for sink strength using transgenic potato plants (Solanum tuberosum L.). Plant J. 7, 97-107.

Conflict of Interest Statement: The authors declare that the research was conducted in the absence of any commercial or financial relationships that could be construed as a potential conflict of interest.

Received: 24 October 2011; accepted: 20 January 2012; published online: 22 February 2012.

Citation: Ferreira SJ and Sonnewald $U$ (2012) The mode of sucrose degradation in potato tubers determines the fate of assimilate utilization. Front. Plant Sci. 3:23. doi: $10.3389 / f p l s .2012 .00023$

This article was submitted to Frontiers in Plant Physiology, a specialty of Frontiers in Plant Science.

Copyright (c) 2012 Ferreira and Sonnewald. This is an open-access article distributed under the terms of the Creative Commons Attribution Non Commercial License, which permits noncommercial use, distribution, and reproduction in other forums, provided the original authors and source are credited. 


\section{APPENDIX}

Table A1 | Phosphorylated intermediates and organic acid content of the different genotypes.

\begin{tabular}{|c|c|c|c|}
\hline Organic acids and phosphorylated intermediates (nmol/g FW) & Desireé & U-IN-1 & U-IN-2 \\
\hline Phosphoenolpyruvate & $22.8 \pm 1.3$ & $26.2 \pm 4.9$ & $40.7 \pm 3.8$ \\
\hline Glucose-6-phosphate & $117.2 \pm 9.1$ & $116.0 \pm 13.5$ & $355.5 \pm 55.2$ \\
\hline Succinate & $83.5 \pm 23.8$ & $32.2 \pm 7.9$ & $108.2 \pm 13.4$ \\
\hline Fumarate & $31.5 \pm 3.8$ & $28.8 \pm 9.1$ & $10.3 \pm 2.3$ \\
\hline 3-Phophoglycerate & $102.1 \pm 9.2$ & $112.6 \pm 8.6$ & $203.1 \pm 7.9$ \\
\hline Trehalose-6-phosphate & $0.9 \pm 0.2$ & $0.3 \pm 0.2$ & $0.5 \pm 0.1$ \\
\hline Sucrose-6-phosphate & $0.5 \pm 0.1$ & $0.4 \pm 0.2$ & $0.1 \pm 0.0$ \\
\hline Fructose-6-phosphate & $22.7 \pm 3.1$ & $47.2 \pm 11.6$ & $126.0 \pm 18.1$ \\
\hline Fructose-1,6-bisphosphate & $0.6 \pm 0.2$ & $0.4 \pm 0.1$ & $1.5 \pm 0.3$ \\
\hline 2-Oxoglutarate & $6.6 \pm 2.6$ & $8.0 \pm 1.4$ & $9.5 \pm 2.1$ \\
\hline Citrate & $7087.9 \pm 720.7$ & $7637.1 \pm 1137.4$ & $8272.5 \pm 420.4$ \\
\hline Malate & $1989.1 \pm 229.5$ & $1757.7 \pm 230.1$ & $3131.7 \pm 29.2$ \\
\hline Inorganic pyrophosphate & $8.4 \pm 2.2$ & $1.8 \pm 0.7$ & $10.6 \pm 0.7$ \\
\hline
\end{tabular}

Results represent the means of three to five replicates $\pm S D$. Bold indicates significance compared to WT $(p \leq 0.05)$.

Table A2 | Amino acid content of the different genotypes.

\begin{tabular}{|c|c|c|c|}
\hline Amino acid (nmol/g FW) & Desireé & U-IN-1 & U-IN-2 \\
\hline Aspartic acid & $490.2 \pm 68.4$ & $378.8 \pm 12.1$ & $1066.0 \pm 39.0$ \\
\hline Glutamic acid & $830.1 \pm 108.8$ & $724.7 \pm 22.0$ & $1130.1 \pm 37.8$ \\
\hline Glutamine & $1594.0 \pm 289.0$ & $1004.4 \pm 116.1$ & $2451.4 \pm 67.5$ \\
\hline Glycine & $147.8 \pm 29.8$ & $95.5 \pm 3.4$ & $145.3 \pm 13.6$ \\
\hline Arginine & $429.0 \pm 81.5$ & $248.3 \pm 20.0$ & $283.3 \pm 7.2$ \\
\hline Alanine & $361.6 \pm 76.2$ & $164.6 \pm 9.3$ & $1099.8 \pm 180.3$ \\
\hline Proline & $71.3 \pm 126.9$ & $55.6 \pm 5.6$ & $69.8 \pm 13.4$ \\
\hline Tyrosine & $1845.4 \pm 9.1$ & $1046.1 \pm 64.5$ & $826.3 \pm 89.5$ \\
\hline Valine & $2365.7 \pm 308.7$ & $1068.5 \pm 31.9$ & $876.3 \pm 41.8$ \\
\hline Phenylalanine & $1222.4 \pm 207.0$ & $688.5 \pm 11.4$ & $1295.0 \pm 98.6$ \\
\hline Total & $21310.9 \pm 4518.9$ & $13861.8 \pm 425.2$ & $19804.1 \pm 1000.2$ \\
\hline
\end{tabular}

Results represent the means of three to four replicates $\pm S D$. Bold indicates significance compared to WT ( $p \leq 0.05)$. 
Table A3 | Major carbohydrates content of the different genotypes.

\begin{tabular}{llll}
\hline Major metabolite $(\boldsymbol{\mu} \mathbf{m o l} / \mathbf{g}$ FW) & Desireé & U-IN-1 & U-IN-2 \\
\hline Starch & $483.1 \pm 73.5$ & $469.8 \pm 28.9$ & $\mathbf{3 9 3 . 3} \pm \mathbf{4 1 . 6}$ \\
Sucrose & $6.8 \pm 1.7$ & $\mathbf{1 . 6} \pm \mathbf{1 . 4}$ & $\mathbf{0 . 2} \pm \mathbf{1 . 2}$ \\
Glucose & $2.0 \pm 1.1$ & $\mathbf{7 . 3} \pm \mathbf{0 . 3}$ & $\mathbf{4 9 . 0 \pm 0 . 2}$ \\
\hline
\end{tabular}

Results represent the means of seven to eight replicates $\pm S D$. Bold indicates significance compared to WT ( $p \leq 0.05)$.

Table A4 | Carotenoid contents of the different genotypes.

\begin{tabular}{llll}
\hline Carotenoids (pmol/g FW) & Desireé & U-IN-1 & U-IN-2 \\
\hline cis-Neoxanthin & $189.5 \pm 13.5$ & $\mathbf{1 4 5 . 3} \pm \mathbf{1 6 . 3}$ & $190.9 \pm 70.3$ \\
Violaxanthin & $943.7 \pm 76.7$ & $\mathbf{1 8 6 . 3} \pm \mathbf{1 5 . 7}$ & $\mathbf{5 6 8 . 2} \pm \mathbf{9 5 . 2}$ \\
Antheraxanthin & $760.0 \pm 91.8$ & $\mathbf{6 2 4 . 2} \pm \mathbf{1 1 . 6}$ & $\mathbf{1 4 0 . 2} \pm \mathbf{8 . 6}$ \\
Lutein & $255.5 \pm 7.9$ & $\mathbf{1 4 0}$ & $231.3 \pm 34.4$ \\
Zeaxanthin & $643.6 \pm 124.8$ & $\mathbf{9 1 2 . 2} \pm \mathbf{6 1 . 2}$ & $\mathbf{1 2 0 0 . 0} \pm \mathbf{1 1 6}$ \\
Beta carotene & $6.8 \pm 3.3$ & $2.9 \pm 0.5$ & $\mathbf{1 9 . 2} \pm \mathbf{1 . 4}$
\end{tabular}

Results represent the means of three to four replicates $\pm S D$. Bold indicates significance compared to WT $(p \leq 0.05)$. 